\title{
Macular ganglion cell layer imaging in preperimetric glaucoma with speckle noise-reduced spectral domain optical coherence tomography.
}

\section{$\operatorname{AUTHOR}(S)$ :}

Nakano, Noriko; Hangai, Masanori; Nakanishi, Hideo; Mori, Satoshi; Nukada, Masayuki; Kotera, Yuriko; Ikeda, Hanako Ohashi; Nakamura, Hajime; Nonaka, Atsushi; Yoshimura, Nagahisa

\section{CITATION:}

Nakano, Noriko ... [et al]. Macular ganglion cell layer imaging in preperimetric glaucoma with speckle noise-reduced spectral domain optical coherence tomography.

Ophthalmology 2011, 118(12): 2414-2426

\section{ISSUE DATE:}

2011-12

\section{URL:}

http://hdl.handle.net/2433/152102

\section{RIGHT:}

(C) 2011 American Academy of Ophthalmology. Published by Elsevier Inc.; This is not the published version. Please cite only the published version.; この論文は出版社版でありません。引用の際には出版社版を ご確認ご利用ください。 
Macular GCL Imaging in Preperimetric Glaucoma. Nakano, et al._Page 1

\section{Macular Ganglion Cell Layer Imaging in Preperimetric Glaucoma with Speckle-Noise-Reduced}

2 Spectral-Domain Optical Coherence Tomography

$3 \quad(133 / 135)$

5 Noriko Nakano, MD, Masanori Hangai, MD, Hideo Nakanishi, MD, Satoshi Mori, MD, Masayuki Nukada, MD, 6 Yuriko Kotera, MD, Hanako Ikeda, MD, Hajime Nakamura, MD, Atsushi Nonaka, MD, Nagahisa Yoshimura, 7 MD

Department of Ophthalmology and Visual Sciences, Kyoto University Graduate School of Medicine, 54

This study was presented at the American Academy of Ophthalmology Annual Meeting, October 2009; San Francisco, California, U.S.A. and received the Best Poster Award.

Proprietary interest statement:

Masanori Hangai is a paid advisory board member for NIDEK CO., LTD., and received consulting fees from Topcon Corporation, and Nagahisa Yoshimura is a paid advisory board member for NIDEK CO., LTD., and is a paid advisory board member for Topcon Corporation.

None of the other authors has a conflict of interest to disclose.

Running head: Macular GCL Imaging in Preperimetric Glaucoma

Support: This research was partially supported by a Grant-in-Aid for Scientific Research (20592038) from the Japan Society for the Promotion of Science (JSPS).

Correspondence to

Masanori Hangai, MD, Department of Ophthalmology and Visual Sciences, Kyoto University Graduate School of Medicine, 54 Kawahara-cho, Shogoin, Sakyo-ku, Kyoto 606-8507, Japan

E-mail: hangai@kuhp.kyoto-u.ac.jp 
Macular GCL Imaging in Preperimetric Glaucoma. Nakano, et al._Page 2

1 Abstract (349/350)

2 Objective: To visualize the macular ganglion cell layer (GCL) and measure its thickness in normal eyes and

3 eyes with preperimetric glaucoma, using speckle-noise-reduced spectral-domain optical coherence

4 tomography (SD-OCT).

5 Design: Retrospective consecutive case series.

6 Participants: Thirty-seven eyes of 37 patients with preperimetric glaucoma and 39 normal eyes of 39

7 volunteers.

8 Methods: Vertical and horizontal SD-OCT B-scan images were acquired with minimal speckle noise by

9 using eye-tracking to obtain and average 50 B-scans at each identical location of interest. B-scan images

10 were manually analyzed for GCL, retinal nerve fiber layer (RNFL), and inner plexiform layer (IPL) shapes

11 and thicknesses in the macula.

12 Main Outcome Measures: Macular GCL images and thickness in normal eyes and in eyes with

13 preperimetric glaucoma.

14 Results: The macular GCL was clearly seen on speckle-noise-reduced SD-OCT images in normal eyes and

15 eyes with preperimetric glaucoma. In each eye with preperimetric glaucoma, thinning of the macular GCL

16 was visually apparent particularly on vertical scans. The mean regional macular GCL was most severely

17 thinned in the inferior perifoveal region, where its thickness was less than $70 \%$ of its normal thickness in 30

$18(81.1 \%)$ of the 37 eyes and less than $50 \%$ of its normal thickness in $13(35.1 \%)$ of the 37 eyes. When the

19 sensitivity and specificity for detecting abnormal thinning (outside the lower limit of $99 \%$ confidence interval

$20[\mathrm{Cl}]$ for the means in the 39 normal eyes) in at least one $0.5-\mathrm{mm}$ segment or sector were compared, the

21 macular GCL on vertical B-scans exhibited higher sensitivity $(81.1 \%)$ than the other layers on vertical 
Macular GCL Imaging in Preperimetric Glaucoma. Nakano, et al._Page 3

1 B-scans $(5.4-59.5 \%, P=0.00075-0.021)$, the macular GCL $(40.5 \%, P=0.00027)$ on horizontal B-scans,

2 the other layers $(5.4 \%-48.6 \%, P<0.00048-0.004)$ on horizontal B-scans, and circumpapillaty RNFL

3 automatically measured on SD-OCT $(54.1 \%, P=0.021)$, and scanning laser polarimetry with variable

4 corneal compensation $(24.3 \% ; P=0.00095)$. All the macular layers on both the vertical and horizontal

$5 \quad$ B-scans and cpRNFL thickness exhibited comparable specificity (91.4-100.0\%, statistically not different).

6 Conclusions: Speckle-noise-reduced SD-OCT imaging allowed clear visualization and measurement of the

$7 \quad$ macular GCL, which was severely thinned in eyes with preperimetric glaucoma. 
Macular GCL Imaging in Preperimetric Glaucoma. Nakano, et al._Page 4

1 In glaucoma, retinal ganglion cell (RGC) loss occurs as a result of damage to the RGC axons within the optic

2 nerve head, and this loss then leads to visual field defects. The results of histological studies of enucleated

3 eyes of patients with glaucoma by Quigley, et al. ${ }^{1}$ and Kerrigan-Baumrind, et al. ${ }^{2}$ and eyes of primates with

4 experimental glaucoma by Harwerth, et al. ${ }^{3}$ indicate that considerable loss of RGCs has occurred by the

5 time alterations in visual function (visual field deficits) are detected clinically. This is consistent with clinical

6 findings that glaucomatous structural changes detectable in the optic nerve head and retinal nerve fiber

7 layer (RNFL) can precede detectable visual field deficits..$^{3-9}$ Thus, direct assessment of the RGCs in patients

8 might be ideal for understanding the damage in eyes with "preperimetric" (before visual field changes)

9 glaucoma. However, studies of RGC loss due to glaucoma in human eyes have been limited to histological

10 approaches using enucleated eyes, because no noninvasive methods are currently available to view

11 individual living RGCs.

Loss of RGCs likely leads to atrophy of the ganglion cell layer (GCL). About $50 \%$ of the RGC somas

13 are concentrated in the macula; subsequently, the macular GCL comprises multilayered RGC somas that

14 generate a thick configuration. Furthermore, the axons of not only macular RGCs but also those of some

peripheral RGCs outside the macula pass through the macular area to reach the optic nerve head. Thus, the macular GCL and RNFL may be promising targets with different characteristics for noninvasive imaging to clinically assess RGC loss. Many previous studies showed that the macula becomes atrophic in eyes with

18 glaucoma when measured using optical imaging modalities. ${ }^{10-19}$ Furthermore, recent studies showed that it

19 is the innermost 3 to 4 layers in the macula that are atrophic in glaucoma, especially in preperimetric

20 glaucoma, when measured using optical coherence tomography (OCT). ${ }^{20-24}$ These results strongly suggest

21 that the macular GCL and RNFL have become atrophic by the time alterations in visual function become 
Macular GCL Imaging in Preperimetric Glaucoma. Nakano, et al._Page 5

1 evident clinically. However, although it is possible to visualize and measure macular RNFL on OCT images, ${ }^{25}$

2 it has been difficult to actually visualize the shape and thickness of the GCL on OCT images because of

3 inability to identify the boundary between the GCL and inner plexiform layer (IPL) with confidence.

Two factors could account for this boundary being obscured on OCT images obtained to date: the

5 image resolution limits of the OCT equipment used and speckle noise. ${ }^{26}$ Because the mean thickness of

6 macular GCL is greater than $30 \mu \mathrm{m},{ }^{21}$ much greater than the axial resolution limit of standard OCT, axial

7 resolution of the equipment used would not fully account for the poor visibility of the GCL boundary in OCT

8 B-scan images. In fact, although ultrahigh-resolution (UHR; $\sim 3 \mu \mathrm{m})$ imaging using a laboratory OCT system

9 improved visualization of the GCL in the macula compared to the standard resolution of time-domain

10 (TD)-OCT systems, the boundary between the GCL and IPL was still unclear, even using UHR-OCT. ${ }^{27,28}$

11 Thus, speckle noise, which is the primary artifact in OCT images and arises from the interference of

12 coherent waves backscattered from nearby scatterers, could be the major factor, ${ }^{29-32}$ and indeed, our and

13 others' studies showed that effective reduction of speckle noise using spectral-domain OCT (SD-OCT)

14 dramatically improves visualization of the boundaries of the GCL. ${ }^{26,31}$

With SD-OCT, B-scan images can be acquired 45 to 130 times faster than with TD-OCT, and when

16 the SD-OCT system includes a 3-dimensional eye-tracking system (Spectralis ${ }^{\mathrm{TM}}$ HRA+OCT, Heidelberg

17 Engineering, Heidelberg, Germany), it is possible to obtain up to 50 to 100 B-scans at the exact same

18 location. Averaging these numerous B-scans obtained at the same location results in sufficient reduction in speckle noise to show the GCL boundary clearly. 
Macular GCL Imaging in Preperimetric Glaucoma. Nakano, et al._Page 6

1 speckle-noise-reduced vertical and horizontal B-scans obtained using the Spectralis SD-OCT in normal eyes

2 and eyes with preperimetric glaucoma.

\section{METHODS}

$5 \quad$ All investigations in this study adhered to the tenets of the Declaration of Helsinki and the study was

6 approved by the Institutional Review Board and Ethics Committee of Kyoto University Graduate School of

$7 \quad$ Medicine. Informed consent for examinations was obtained from all participants.

9 SD-OCT at Kyoto University Hospital (Kyoto, Japan) between July 14, 2008, and June 15, 2009, and

10 volunteers with healthy eyes. Preperimetric glaucoma was defined as evident glaucomatous appearance of

11 the optic nerve head in eyes with normal visual fields. Volunteer eyes were categorized as normal if they had 12 an intraocular pressure (IOP) of $21 \mathrm{mmHg}$ or less, a normal-appearing optic disc head, and normal results 13 with visual field testing.

Exclusion criteria for both groups were best-corrected visual acuity (BCVA) worse than 20/20 in the

15 Snellen equivalent, equivalent refractive error of more than 3 diopters $(D)$ or $<-6 D$, unreliable visual field results (fixation loss $>20 \%$, false-positive $>15 \%$ or false-negative $>33 \%$ ) on standard automated perimetry

17 (SAP) using the Humphrey 24-2 Swedish Interactive Threshold Algorithm (SITA) (HFA, Carl Zeiss Meditec, 18 Dublin, CA), evidence of vitreoretinal disease, or diabetes mellitus or any other systemic disease that might 19 affect the eye. 
Macular GCL Imaging in Preperimetric Glaucoma. Nakano, et al._Page 7

1 slit-lamp examinations, IOP measurements using a Goldmann applanation tonometer, standard automated

2 perimetry (SAP), Heidelberg Retina Tomography II (HRT II, Heidelberg Engineering), scanning laser

3 polarimetry with variable corneal compensation (GDx-VCC, Carl Zeiss Meditec), dilated funduscopy, stereo

4 disc photography using a 3-Dx simultaneous stereo disc camera (Nidek, Gamagori, Japan), and Spectralis

$5 \quad$ SD-OCT.

6

$7 \quad$ Visual Field Testing

8 Reliable visual field test results (fixation loss $\leq 20 \%$; false positives $\leq 15 \%$ and false negatives $\leq 33 \%$ ) on

9 standard automated perimetry (SAP), using the Humphrey 24-2 Swedish Interactive Threshold Algorithm

10 (SITA) (HFA, Carl Zeiss Meditec) were used. Visual field defects caused by glaucoma were defined, on the

11 basis of SAP results, as (1) abnormal range on the glaucoma hemifield test or (2) a pattern standard

12 deviation (PSD) of $<5 \%$ from the normal reference. Results were confirmed by 2 consecutive tests.

14 Optic Disc Evaluation

15 The appearance of the optic nerve head on fundus photographs, including the stereoscopic photographs,

16 was independently evaluated by 3 glaucoma specialists $(\mathrm{MH}, \mathrm{HOI}$, and $\mathrm{AN})$ who were masked to all other

17 data about the eyes. Images of each eye were displayed on a monitor for each eye from both normal and

18 preperimetric glaucoma candidates. Glaucomatous optic nerve head appearance was defined as the

19 presence of diffuse or localized rim thinning, disc hemorrhage, or a vertical cup-to-disc ratio of 0.2 or greater

20 than that in the fellow eye. If the decisions of all 3 examiners were not in agreement, consensus was

21 reached by group review and discussion of the fundus color photographs and stereophotographs. 
Macular GCL Imaging in Preperimetric Glaucoma. Nakano, et al._Page 8

\section{Speckle-Noise-Reduced SD-OCT}

3 Horizontal and vertical speckle-noise-reduced SD-OCT imaging centered on the fovea centralis was

4 performed using the Spectralis ${ }^{\mathrm{TM}} \mathrm{HRA}+\mathrm{OCT}^{26}$ Each A-scan of this instrument had a depth of $2 \mathrm{~mm}$ and

5 comprised 512 pixels, providing a digital depth sampling of $3.9 \mu \mathrm{m}$ per pixel. Each B-scan for this study

6 spanned $30^{\circ}$ (approximately $9 \mathrm{~mm}$ ) and consisted of 1,536 A-scans (acquired at a rate of 40,000 per

7 second), providing a digital transverse sampling resolution of $5 \mu \mathrm{m}$ per pixel. The combination of

8 high-resolution scanning laser imaging of the retina and SD-OCT enables real-time 3-dimensional tracking

9 of eye movements and real-time averaging of multiple B-scans acquired at each identical location of interest

10 on the retina to reduce speckle noise. Radial scans centered at the fovea or vertical serial scans were also

11 obtained.

13 Hand Delineation of Retinal Layer Boundaries and Thickness Measurements

14 The boundaries of the RNFL (vitreoretinal interface and the border between RNFL and GCL), GCL (the

15 borders between RNFL and GCL and between GCL and IPL), and inner plexiform layer (IPL) (the borders

16 between GCL and IPL and between IPL and the inner nuclear layer) were drawn on SD-OCT B-scan images

17 by 2 independent delineators at the Kyoto OCT Reading Center ${ }^{33}$ who were masked to all clinical

18 information using custom-made functions written into the standard built-in Spectralis ${ }^{\mathrm{TM}} \mathrm{HRA}+\mathrm{OCT}$ software

19 by Heidelberg engineers. The delineators marked areas of "fringe washout" where shadows from blood

20 vessels prevented visualization of underlying structures and where retinal layers were disrupted or deformed

21 by blood vessels or their shadows. Where the structure of intraretinal layers was disrupted in this way, the 
Macular GCL Imaging in Preperimetric Glaucoma. Nakano, et al._Page 9

1 delineators connected by a straight line the end points of each boundary line on either side of the disruption.

\section{Inter-Delineator Variability Assessment}

4 To assess inter-delineator variability, raw results obtained independently by 2 delineators were compared. To

5 assess the accuracy of the delineations of each delineator for each eye, a third independent observer (NN),

6 who was also masked to clinical information about the eyes, joined the group review with the 2 delineators to

7 check the boundary lines. If both the 2 delineators' results were judged to be accurate by group review, one

8 of the results was randomly selected for use in analysis. If one of the 2 delineators' results was judged to be

9 inaccurate by group review, the other boundary line was selected for use in analysis. If both the 2

10 delineators' results were judged inaccurate, the review group decided how they should be corrected; once

11 corrected, 1 result was arbitrarily chosen for inclusion. To visualize inter-delineator variability, we

12 superimposed the mean raw GCL thickness data of the 2 delineators in a graph. To display the symmetry

13 along the horizontal and vertical B-scans, we also superimposed the mean arbitrated GCL thickness data in

14 the nasal-temporal and superior-inferior hemispheres.

After manual delineation of retinal layer boundaries, Spectralis software was used to measure

thicknesses of each layer by calculating the distance in pixels between that layer's inner and outer boundary

lines and converting the result to micrometers. The custom software displays a single thickness value on each A-scan (e.g., every $0.5-\mathrm{mm}$ point from the foveal center) and a mean thickness value that is averaged within each defined region (macula, superior, and inferior hemisphere, parafovea [0.5-1.5 $\mathrm{mm}$ from the foveal center] and perifovea [1.5-3.0 $\mathrm{mm}$ from the foveal center], and every $0.5-\mathrm{mm}$ segment). 
Macular GCL Imaging in Preperimetric Glaucoma. Nakano, et al._Page 10

1 for normal eyes in individual eyes, after checking normal distribution of thickness values in normal eyes, we

2 computed $95 \%$ and $99 \%$ confidence intervals (Cls) for the mean thickness of each layer in the normal study

3 eyes. Thickness measurements in the eyes with preperimetric glaucoma were then categorized on the basis

4 of thickness measurements obtained for the normal eyes: "normal," measurement within the $95 \% \mathrm{Cl}$ values

5 for the mean thickness in normal eyes; "borderline thickened," between the upper 95\% and upper $99 \% \mathrm{Cl}$

6 limit for the mean in normal eyes; "borderline thin," between the lower 95\% and lower 99\% Cl limit for the

7 mean in normal eyes; "abnormally thick," above the upper $99 \% \mathrm{Cl}$ limit for the mean in normal eyes; or

8 "abnormally thin," below the lower $99 \%$ Cl limit for the mean in normal eyes.

10 Automated Circumpapillary RNFL Thickness Measurements

11 GDx-VCC and Spectralis were used to assess circumpapillary RNFL (cpRNFL) thickness. For both

12 instruments, cpRNFL thickness was automatically measured using built-in software, and abnormal thinning

13 was displayed on the basis of the Cls of the instrumental normative databases. For cpRNFL analysis on

14 Spectralis, we also computed $99 \%$ Cls for the mean thickness of cpRNFL in the 39 normal eyes.

16 Sensitivity and Specificity

17 We calculated sensitivity and specificity for detecting abnormal thinning (outside the lower $99 \% \mathrm{Cl}$ limit for

18 the mean of the 39 normal eyes) of each macular layer in at least one $0.5-\mathrm{mm}$ segment of the macula on

19 vertical and horizontal B-scans. We also calculated sensitivity and specificity for the detection of abnormal

20 cpRNFL thinning. In Spectralis, cpRNFL was defined as "abnormal" when the mean thickness of the whole

$21 \mathrm{cpRNFL}$ or of at least one sector of the Spectralis sector map was abnormally thin (outside the lower $99 \% \mathrm{Cl}$ 
Macular GCL Imaging in Preperimetric Glaucoma. Nakano, et al._Page 11

1 limit for the mean of the instrumental normative database or the 39 normal eyes). In GDx-VCC, cpRNFL was

2 defined as "abnormal" when the mean values of at least one thickness parameters among "NFI," "TSNIT

3 average," "superior average," "inferior average," or "TSNIT Std. Dev." was abnormal (outside the lower 99\%

$4 \mathrm{Cl}$ limit for the mean of the instrumental normative database). An additional group of 35 normal eyes were

$5 \quad$ used to calculate specificity.

\section{$7 \quad$ Statistical Analyses}

8 Differences in the age and spherical equivalent of refractive errors between normal and preperimetric

9 glaucoma groups were compared using unpaired $t$-tests. Differences in gender between the normal and

10 preperimetric glaucoma groups were compared using Fisher's exact test. McNemar's test was used to

11 compare the sensitivity and specificity for detecting abnormal thinning of macular layer and $\mathrm{cpRNFL}$

12 thickness. Statistical analyses were performed using PASW Statics version 17.0 (SPSS Inc., Chicago, IL).

13 The level of statistical significance was set at $P<0.05$.

RESULTS

16 Among the 49 candidate eyes that were selected on the basis of the inclusion/exclusion criteria for the

17 preperimetric group, 37 eyes were judged to have apparent glaucomatous abnormalities, such as

18 neuroretinal rim thinning associated with wedge-shaped localized nerve fiber layer defects (NFLDs; 35

19 eyes), disc hemorrhages ( 11 eyes), and both (9 eyes). Evaluation findings of the 3 specialists were

20 unanimous for all the 37 eyes. The evaluation findings of the 3 specialists did not agree in 6 eyes out of the

21 other 10 candidate eyes with preperimetric glaucoma and in 11 of the 44 normal-candidate eyes. The 3 
Macular GCL Imaging in Preperimetric Glaucoma. Nakano, et al._Page 12

1 glaucoma specialists made final decisions for these eyes by group discussion. Even after group discussion,

2 the specialists could not reach agreement regarding 2 candidate eyes with preperimetric glaucoma and 2

3 normal-candidate eyes; these were excluded from analysis. A total of 47 eyes of 47 patients were identified

4 as having preperimetric glaucoma, and 42 eyes from 42 volunteers were identified as normal. After

$5 \quad$ excluding eyes with poor-quality speckle-noise-reduced B-scans, the remaining 37 eyes of 37 patients

6 constituted the preperimetric glaucoma group, and 39 eyes of 39 subjects constituted the normal group for

7 comparison. In the preperimetric glaucoma group, 29 eyes were judged to have neuroretinal rim thinning

8 associated with wedge-shaped localized NFLDs, 9 with disc hemorrhages, and 7 with both. Six eyes were

9 judged to have a vertical cup-to-disc ratio of 0.2 or greater compared to that of the fellow eye; however, all

10 these eyes had disc hemorrhages or neuroretinal rim thinning associated with NFLDs.

The 37 patients with preperimetric glaucoma (24 men and 13 women) ranged in age from 30 to 73

12 years (mean \pm standard deviation $[S D], 58.0 \pm 9.9$ years) and the spherical equivalent of the refractive errors

13 of the 37 eyes with preperimetric glaucoma ranged from -5.5 to $+2.0 \mathrm{D}$ (mean $\pm S D,-1.0 \pm 2.3 \mathrm{D}$ ). The 39

14 volunteers (19 men and 20 women) were used to determine abnormal thinning of macular and cpRNFL

15 parameters and to compute $95 \%$ and $99 \%$ Cls; they ranged in age from 22 to 70 years (mean \pm SD, $56.0 \pm$

1613.0 years) and the spherical equivalent of the refractive errors of the 39 normal eyes ranged from -5.5 to

$17+1.0 \mathrm{D}$ (mean $\pm \mathrm{SD},-1.3 \pm 2.1 \mathrm{D})$. There were no statistically significant differences in gender, age or

18 spherical equivalent of refractive errors between patient and normal groups $(P=0.346,0.191$, and 0.737 ,

19 respectively). 
Macular GCL Imaging in Preperimetric Glaucoma. Nakano, et al._Page 13

1 discussion or poor-quality speckle-noise-reduced B-scans. The volunteers' age ranged from 34.2-76.4

2 years (mean \pm SD, $57.4 \pm 10.7$ years), and the spherical equivalent of the refractive errors ranged from

$3-5.5-2.0 \mathrm{D}(-1.4 \pm 2.1 \mathrm{D})$. There were no statistically significant differences with respect to gender, age, or

4 the spherical equivalent of refractive errors between the patients and the other normal groups $(P=0.339$,

$5 \quad 0.352$, and 0.737 , respectively).

The disc areas of the study eyes in the preperimetric, first normal, and second normal groups

7

$9 \quad 0.11$, and $0.99 \pm 0.12$, respectively.

\section{Visualization of Retinal Layer Boundaries}

12 On speckle-noise-reduced SD-OCT images, the boundary of each retinal layer, including the boundary

13 between the GCL and IPL, was clearly distinguishable (Fig 1). The boundary became more clear as the

14 number of B-scans averaged increased (Fig 2), but there was little difference between averages of 50 and

$15100 \mathrm{~B}$-scans, so images generated by averaging $50 \mathrm{~B}$-scans were used for this study.

17 Macular GCL Appearance and Thickness in Normal Eyes

18 In normal eyes, the GCL in the macula was seen as a well-demarcated hyporeflective layer that had a fairly

19 symmetrical shape across the central fovea (Figs 1 and 2 ). When the mean macular GCL thickness was

20 compared at every $0.5-\mathrm{mm}$ point from the foveal center, however, the mean macular GCL thickness in the 39

21 normal eyes appeared to be more symmetrical on either side of the fovea on vertical than on horizontal 
Macular GCL Imaging in Preperimetric Glaucoma. Nakano, et al._Page 14

1 B-scans (Fig 3).

On vertical B-scans in normal eyes, as distance from the center of the fovea increased, macular

3 GCL thickness increased rapidly to a peak between 0.73 and $1.40 \mathrm{~mm}$ superior to the foveal center (mean \pm

$4 \mathrm{SD}, 0.95 \pm 0.18 \mathrm{~mm}$ ) and from 0.70 to $1.20 \mathrm{~mm}$ inferior to the foveal center (mean $\pm \mathrm{SD}, 0.87 \pm 0.43 \mathrm{~mm}$ )

5 (Fig 3). Peak macular GCL thickness ranged from 43 to $80 \mu \mathrm{m}$ (mean $\pm \mathrm{SD}, 64.5 \pm 7.9 \mu \mathrm{m}$ ) in the superior

6 retinal hemisphere and from 40 to $81 \mu \mathrm{m}$ (mean $\pm \mathrm{SD}, 66.6 \pm 7.4 \mu \mathrm{m}$ ) in the inferior hemisphere. Macular

$7 \quad$ GCL thickness gradually decreased with greater distance from the fovea (Fig 3). On horizontal B-scans,

8 peak macular GCL thickness was located slightly more distant from the foveal center than on vertical

9 B-scans: the nasal peak was 0.9 to $1.9 \mathrm{~mm}(1.29 \pm 0.20 \mathrm{~mm})$ from the center of the fovea and the temporal

10 peak was 0.8 to $1.6 \mathrm{~mm}(1.24 \pm 0.18 \mathrm{~mm})$ from the foveal center (Fig 3). Macular GCL thickness decreased

11 more rapidly from its peak to the periphery on vertical compared to horizontal B-scans.

The superior and inferior macular GCL thicknesses showed statistically significant differences at

$1.75,2.00,2.25$, and $3 \mathrm{~mm}$ from the central fovea; the mean differences were $4.7,6.3,5.7$, and $2.6 \mu \mathrm{m}$,

respectively, all of which were less than the axial-resolution $(7 \mu \mathrm{m})$ of Spectralis. The nasal and temporal

thickness showed statistically significant differences at 1.00, 1.25, 1.50,1.75, 2.00, and $2.25 \mathrm{~mm}$ from the

central fovea; the differences were $5.3,6.5,7.5,8.6,7.5,4.2 \mu \mathrm{m}$, respectively, and more than the

axial-resolution of $7 \mu \mathrm{m}$ of Spectralis at $1.5,1.75$, and $2.0 \mathrm{~mm}$ from the central fovea. Therefore, we compared the nasal-temporal differences with the measurement variability induced by the 2 delineators at thickness between 2 delineators with regard to nasal and temporal thickness differences were -4.3 to 2.6 
Macular GCL Imaging in Preperimetric Glaucoma. Nakano, et al._Page 15

1 thicknesses were 7.5, 8.6, and $7.5 \mu \mathrm{m}$, respectively (Fig 3). Thus, at $1,1.75$, and $2.0 \mathrm{~mm}$ from the central

2 fovea, the mean differences between the nasal and temporal thicknesses were outside the limits of $95 \% \mathrm{Cls}$

3 for the mean differences between 2 delineators when measuring nasal and temporal thickness.

5 Mean Macular Retinal Layer Thicknesses on Vertical and Horizontal B-Scans for Preperimetric

\section{Glaucoma}

$7 \quad$ When the mean macular GCL thickness was compared between normal eyes and eyes with preperimetric

8 glaucoma at every $0.5-\mathrm{mm}$ point from the foveal center (Fig 4), the mean GCL thickness was significantly

9 thinner in eyes with preperimetric glaucoma than in normal eyes at almost all distances on vertical and

10 horizontal B-scans (inferiorly at $0.5-4 \mathrm{~mm}$, superiorly at $0.5-1.0$, and $1.5-4 \mathrm{~mm}$, nasally at $0.5-3.25 \mathrm{~mm}$,

11 and temporally at $0.5-3.75 \mathrm{~mm}$ from the foveal center). However, the thickness difference between the

12 groups was greater than the axial-resolution $(7 \mu \mathrm{m})$ of Spectralis inferiorly $(0.75-2.75 \mathrm{~mm})$ and temporally

$13(0.75-2.75 \mathrm{~mm})$ from the foveal center, and superiorly (only at $0.75,1.0$, and $1.75 \mathrm{~mm}$ ), but nasally not

14 greater at any segment.

When mean thicknesses of each inner retinal layer (RNFL, GCL, and IPL) averaged within defined

regions on vertical and horizontal B-scans were compared, eyes with preperimetric glaucoma had significant

17 thinning of the RNFL, the GCL, and RNFL + GCL + IPL (termed the "RGC complex") compared to normal

18 eyes in the whole region, in both the inferior and superior hemispheres and at all distances from the center

19 of the fovea $(P<0.0001$ to $P=0.027$, Table 1$)$. When viewed on horizontal B-scans, eyes with preperimetric

20 glaucoma exhibited significant thinning of the RNFL, GCL, and RGC complex compared to normal eyes in

21 the whole macula, in both inferior and superior hemispheres, and at all distances from the center of the 
Macular GCL Imaging in Preperimetric Glaucoma. Nakano, et al._Page 16

1 fovea except for the parafoveal regions for RNFL and the nasal parafoveal region for the RGC complex $(P<$

20.0001 to $P=0.043$, Table 1). Eyes with preperimetric glaucoma did not have significant thinning of the

3 macular IPL in the vertical or horizontal B-scans (Table 1).

Table 1 also shows the number and percentage of eyes with abnormal thinning in each layer at

each macular region in eyes with preperimetric glaucoma, which varied according to the layer, region, and

vertical/horizontal B-scan. The percentage of eyes with abnormal thinning appears to be larger roughly in

7 the order of GCL $>$ RGC complex $>$ RNFL $>$ IPL on vertical B-scans and GCL $=R G C$ complex $>$ RNFL $>$ IPL

on horizontal B-scans. With regard to the macular GCL, the percentage of eyes with abnormal thinning appears to be larger in the order of "inferior" $(51.4 \%)>$ "superior" $(18.9 \%)=$ "temporal" $(18.9 \%)>$ "nasal" (8.1\%) hemisphere and "perifovea" $(67.6 \%)$ > "parafovea" $(35.1 \%)$ on the vertical B-scans and "perifovea" $(16.2 \%)$ < "parafovea" $(29.7 \%)$ on the horizontal B-scans. In addition, the percentage of eyes with abnormal macular GCL thinning appears to be greater in vertical B-scans (10.8\%-51.4\%) than in horizontal

Figure 5 shows the distribution of percent differences in regional mean thicknesses of the RNFL,

GCL, IPL, and RGC complex (RNFL + GCL + IPL) in vertical and horizontal B-scans in eyes with

preperimetric glaucoma compared to normal eyes. The mean macular GCL, RNFL, and RGC complex were most severely thinned in the inferior perifovea, where mean macular GCL thickness was less than $70 \%$ of normal in $30(81.1 \%)$ of the 37 eyes and less than $50 \%(49.8 \%$ to $21.2 \%)$ of normal in $13(35.1 \%)$ of the 37 eyes (Fig 5A,B), the macular RNFL thickness less than $70 \%$ of normal in $13(35.1 \%)$ (Fig 5C,D), and the 20 macular RGC complex thickness less than $80 \%$ of normal in $14(37.8 \%)$ (Fig $5 \mathrm{G}, \mathrm{H})$. In the inferior perifoveal 
Macular GCL Imaging in Preperimetric Glaucoma. Nakano, et al._Page 17

\section{Sensitivity and Specificity for Detecting Abnormal Thinning}

3 The sensitivity and specificity for detecting abnormal thinning (outside the lower $99 \% \mathrm{Cl}$ limit) in each

4 macular layer thickness on vertical and horizontal B-scans, and in cpRNFL thickness were compared (Table

5 2). The macular GCL on vertical B-scans exhibited higher sensitivity $(81.1 \%)$ than the other layers on vertical

6 B-scans $(5.4-59.5 \%, P=0.00075-0.021)$, the macular GCL $(40.5 \%, P=0.00027)$ on horizontal B-scans,

7 and the other layers $(5.4 \%-48.6 \%, P<0.00048-0.004)$ on horizontal B-scans. The sensitivity for detecting

8 abnormal macular GCL thinning was only slightly higher (83.8\%) when eyes with abnormal thinning were

9 counted either on vertical or horizontal B-scan; this sensitivity was higher than that for the RNFL $(56.8 \%, P=$

$100.0060)$ and RGC complex $(70.3 \% ; P=0.13)$ counted on vertical and horizontal B-scans; the difference

11 regarding the RGC complex was not statistically significant. All the layers on both the vertical and horizontal

12 B-scans exhibited comparable specificity (94.3-100.0\%, statistically not different). The sensitivity for

13 detecting abnormal thinning in the macular GCL either on the vertical or horizontal B-scan was significantly

14 higher than that for cpRNFL thickness measured on Spectralis $(43.2 \%, P=0.0070$ [calculated using the

15 instrumental normative database]; $54.1 \%, P=0.0010$ [calculated using the data of 39 normal eyes]) and

16 GDx-VCC (24.3\%; $P=0.00040)$. The sensitivity for detecting abnormal thinning in the macular RGC

17 complex either on vertical or horizontal B-scan was significantly higher than that for cpRNFL thickness

18 assessed on Spectralis by using the instrumental normative database $(P=0.006)$ and GDx-VCC $(P=$

19 0.00076); however, it was not significantly different from the cpRNFL thickness assessed on Spectralis by

20 using the data from 39 normal eyes $(P=0.109)$. Specificity was comparable (statistically not different)

21 between the macular parameters (91.4-100.0\%) on the vertical or horizontal B-scans as well as cpRNFL 
Macular GCL Imaging in Preperimetric Glaucoma. Nakano, et al._Page 18

1 thickness (94.3-97.1\%) measured on both instruments.

\section{Case Report}

4 In many eyes with preperimetric glaucoma, local GCL thinning was apparent on visual examination of

5 SD-OCT B-scans, particularly on vertical B-scans (Figs 6-8).

7 in her left eye was $14-16 \mathrm{mmHg}$ during the first 3 visits. On photographs, the optic disc showed thinning of

8 the inferotemporal neuroretinal rim and a corresponding localized wedge-shaped RNFL defect (Fig 6A,B).

9 The patient had a normal pattern deviation map (Fig 6D) on SAP and normal glaucoma hemifield test, and

10 PSD results. On 9-mm length radial SD-OCT B-scans, local GCL atrophy (Fig 6E,K,L) was evident

11 particularly in the inferior hemisphere when compared to an equivalent area in the superior hemisphere (Fig

$126 \mathrm{E}, \mathrm{I}, \mathrm{K})$. Thinning of the RNFL was associated with the thinning of the GCL. The RNFL in the inferior

13 hemisphere area was borderline thinned (outside the lower $95 \% \mathrm{Cl}$ limit in 39 normal eyes) from -2.0 to -3.0

$14 \mathrm{~mm}$ (Fig 7B). The GCL in the inferior hemisphere area was abnormally thinned from -1.5 to $-3.0 \mathrm{~mm}$ on the

15 vertical B-scan (Fig 7C). Here, the thinning of the macular GCL appeared to be more abrupt and severe. The

16 IPL thickness remained within the 95\% normal Cl (Fig 7D). Changes in thickness of the RGC complex (Fig

17 7E) appeared to essentially mirror changes in GCL thickness (Fig 7C). The mean and sectorial cpRNFL

18 thickness were normal (Fig 7G).

\section{DISCUSSION}

21 Glaucoma causes loss of RGCs, which is thought to lead to GCL thinning. To date, however, the GCL has 
Macular GCL Imaging in Preperimetric Glaucoma. Nakano, et al._Page 19

1 been difficult to see clearly on single OCT scans, even on ultrahigh-resolution images, ${ }^{27,28}$ because of

2 speckle noise in B-scan images. ${ }^{26,29-32}$ This study is the first, to our knowledge, to show that the macular

3 GCL in human eyes can be visualized and measured using the speckle-noise-reduction method with

4 SD-OCT B-scan images. We used this method to test the hypotheses that the macular GCL is diminished in

5 preperimetric glaucoma and showed that the macular GCL was diminished on vertical and horizontal

6 speckle-noise-reduced B-scans through the fovea; both the GCL and RNFL were diminished, but the GCL

$7 \quad$ was substantially more diminished.

In normal eyes in our study, on both vertical and horizontal speckle-noise-reduced SD-OCT images,

9 the macular GCL appeared to have the appearance of a fairly symmetric configuration with peak thickness in

10 the central parafoveal region (about $1 \mathrm{~mm}$ from the center of the fovea). These results are consistent with

11 reports on RGC densities in enucleated human eyes. ${ }^{34-36}$ Mean macular GCL thickness topography curves

12 on vertical and horizontal scans in our study exactly matched fairly symmetric RGC density topography

13 curves shown on vertical and horizontal meridians in enucleated eyes. ${ }^{36}$

In our study, however, the mean macular GCL thicknesses in normal eyes showed differences of the central fovea, but did not show differences of more than $7 \mu \mathrm{m}$ at any distance in the inferior and superior hemispheres. In addition, the nasal and temporal thickness differences of these distances were outside the manual measurement variability limit between delineators. Thus, in the current imaging and measurement settings, the nasal-temporal difference in macular GCL thickness in normal eyes appears to be an actual difference (actual asymmetry) but the superior-inferior thickness difference does not. 
Macular GCL Imaging in Preperimetric Glaucoma. Nakano, et al._Page 20

1 visually apparent thinning of the macular GCL, particularly when compared to an equivalent area in the

2 opposite hemisphere and also when compared to the GCL configuration of normal eyes. The macular GCL

3 thickness measurements confirmed that the GCL was abnormally thin as compared to the normal mean

4 thickness in $30(81.1 \%)$ of our 37 study eyes at least in one $0.5-\mathrm{mm}$ segment. In addition, although the mean

5 macular GCL thickness only exhibited a small difference ( $81.8 \%$ of the normal means), the mean regional

6 macular GCL on vertical B-scans in eyes with preperimetric glaucoma was $57.8 \%$ of the normal means in

7 the inferior perifovea; it was thinned in the inferior perifovea to less than $70 \%$ of the normal means in 30

$8(81.1 \%)$ of the 37 eyes and to less than $50 \%$ of the normal means in $13(35.1 \%)$ eyes. Thus, when we

9 observed macular GCL thinning on vertical B-scans in particular, the macular GCL thinning in the

10 preperimetric glaucoma appeared to occur in local regions; such local thinning would not cause severe

11 thinning in the mean GCL in the macula. These observations of macular GCL thinning in eyes with

12 preperimetric glaucoma appears to be consistent with previous histological studies with enucleated eyes of

13 patients with glaucoma, which suggested that considerable loss of macular RGCs in local regions

14 corresponding to the visual field test points occurs before visual field defects are detected. ${ }^{1}$ Thus,

15 speckle-noise-reduction on SD-OCT images may be useful for visualizing and measuring preperimetric

16 RGC damage clinically in patients.

In previous studies that used OCT to study macular RCG structures in eyes with glaucoma, Stratus

18 OCT with originally developed automated measurement software or SD-OCT with commercially available

19 automated measurement software ${ }^{20-23}$ was used to measure thickness of the RGC complex of the macula,

20 including the RNFL, GCL, and IPL (termed ganglion cell complex, GCC). These studies consistently showed

21 that the RGC complex was more severely thinned than the total retina in eyes with glaucoma, and of these 
Macular GCL Imaging in Preperimetric Glaucoma. Nakano, et al.-Page 21

1 studies, 2 that were by Tan et al. ${ }^{21,22}$ showed that the RGC complex was significantly thin in eyes with

2 preperimetric glaucoma compared to in normal eyes. However, the configuration of the GCL and its thinning

3 as a result of glaucoma was not clearly visualized in these previous studies. Our study showed that each

4 single layer in the macula was clearly distinguished and measurable, and that the macular GCL and RNFL

$5 \quad$ but not the IPL were significantly thinned in eyes with preperimetric glaucoma compared to normal eyes.

6 Thus, our results were basically consistent with those of these previous studies, but provided more details

7 about damages occurring in the individual layers within the RGC complex in preperimetric glaucoma.

RGC damage as a single parameter. The feasibility of the automated measurement of the RGC complex

10 with low variability underlies its advantage.$^{21,34}$ However, a disadvantage is that the glaucomatous damage

11 of each layer, such as the RNFL, GCL, and IPL, cannot be determined. Because of the anatomical

12 characteristics of the macula, there are differing configurations and symmetry, particularly between the

13 macular RNFL, and GCL_especially the axons of the macular RGCs and the axons of some peripheral

14 RGCs outside the macula merge to pass through the macular area, forming the macular RNFL. Accordingly,

15 morphological changes of the macula caused by the loss of RGCs in glaucoma are not necessarily identical

16 in each layer or region. In fact, our findings suggest that abnormal thinning of each macular layer possibly

17 varies by layer, region, and vertical/horizontal B-scan. Thus, it appears that it is important to know the extent

18 of glaucomatous damage to each layer, such as GCL and RNFL. Our knowledge of preperimetric

19 glaucomatous RGC loss is based on previously reported histopathological studies of the retina and optic

20 nerve using enucleated patients eyes. ${ }^{1,2}$ It is difficult to use such histopathological methods for a large scale 
Macular GCL Imaging in Preperimetric Glaucoma. Nakano, et al._Page 22

1 individual RGCs, speckle-noise-reduction in SD-OCT imaging provides a unique opportunity for

2 non-invasively observing and assessing damages of RGC somas, and axons as abnormal thinning of the

3 macular GCL and RNFL, which will be able to be used for future clinical research in a large scale.

In our study, we could not fully assess the glaucoma discriminating ability of macular GCL because

5 automated measurement of each layer was not available in the instrument used, and because our analysis

6 did not include the entire macular area. Although macular GCL exhibited highest sensitivity for detecting

7 preperimetric glaucomatous abnormalities, it remains unclear whether the measurement of a single GCL

8 layer can discriminate glaucoma better than the measurement of the RGC complex. In our preliminary

9 experiments conducted by using original $\mathrm{Cl}$ maps on the basis of 39 normal eyes, the macular GCL either

10 exhibited higher sensitivity than the other macular layers including the macular RGC complex, although the

11 difference regarding the macular RGC complex was not statistically supported in our subjects. Macular GCL

12 exhibited higher sensitivity than cpRNFL thickness as assessed on Spectralis by using the same normal

13 data, whereas we did not detect significantly higher sensitivity in the macular RGC complex compared to the

14 cpRNFL thickness assessed by using the same normal data. It was previously shown that selective

measurement of the macular RGC complex has better ability to discriminate glaucoma than measurement of

16 the total retinal thickness in the macula. ${ }^{20-25,34}$ This is attributed to the fact that the total retina includes the

17 outer retinal layers that are minimally diminished in glaucoma (if any), whereas the macular RGC complex

18 does not. ${ }^{22}$ Similarly, inclusion of macular IPL, which did not exhibit significant thinning on vertical or

19 horizontal B-scans in our study, potentially decreases the sensitivity of the macular RGC complex for

20 detecting glaucomatous changes. Thus, although our study was performed manually, the results encourage

21 the development of an automated segmentation algorithm for dense B-scans over the whole macula to 
Macular GCL Imaging in Preperimetric Glaucoma. Nakano, et al._Page 23

1 determine whether selective measurement of GCL may have comparable or better glaucoma-discriminating

2 ability than known OCT parameters such as $\mathrm{CPRNFL}$ and RGC complex.

The results of this study may raise the question, "How can SAP results remain normal after the GCL

4 has become abnormally thin?" One possible explanation is that there may be sufficient redundancy in the

5 visual system of RGCs in the normal macula whose function, at least as measured by SAP, remains normal

6 despite considerable loss of RGCs. ${ }^{1-3}$ Another possible explanation is that there is some loss of function, but

7 it is not identified by the SAP 24-2 program because of the relatively low spatial resolution of test points; test

8 points are about 5 degrees apart, representing a distance of almost $1.25 \mathrm{~mm}$ on the retina; hence, thinning

9 of the macular GCL limited to areas between the SAP test points might not be detected by SAP. Further

10 studies using short-wavelength automated perimetry, frequency-doubling technology perimetry, and 10-2

11 SAP may clarify this question.

A limitation of this pilot study is that thickness measurements were performed only on vertical and

13 horizontal B-scan images, which do not show the entire macula; these images show only certain aspects of

14 macular GCL morphology. Despite this limitation, our study demonstrated that speckle-noise-reduction in

15 SD-OCT imaging can be used to clinically visualize and measure the macular GCL in human eyes. On the

16 speckle-noise-reduced SD-OCT images, the macular GCL in eyes with preperimetric glaucoma showed

17 visually apparent and quantitatively abnormal thinning as compared to normal eyes. Macular GCL imaging

18 using speckle-noise-reduction of SD-OCT B-scans may be potentially useful for facilitating understanding of 19 glaucomatous structural damages of the macula. 
Macular GCL Imaging in Preperimetric Glaucoma. Nakano, et al._Page 24

\section{REFERENCES}

2 1. Quigley HA, Dunkelberger GR, Green WR. Retinal ganglion cell atrophy correlated with automated perimetry in human eyes with glaucoma. Am J Ophthalmol 1989;107:453-64.

2. Kerrigan-Baumrind LA, Quigley HA, Pease ME, et al. Number of ganglion cells in glaucoma eyes compared with threshold visual field tests in the same persons. Invest Ophthalmol Vis Sci $2000 ; 41: 741-8$.

3. Harwerth RS, Carter-Dawson L, Shen F, et al. Ganglion cell losses underlying visual field defects from experimental glaucoma. Invest Ophthalmol Vis Sci $1999 ; 40: 2242-50$.

4. Hoyt WF, Newman NM. The earliest observable defect in glaucoma? Lancet 1972;1:692-3.

5. Sommer A, Pollack I, Maumenee AE. Optic disc parameters and onset of glaucomatous field loss. I. Methods and progressive changes in disc morphology. Arch Ophthalmol 1979;97:1444-8.

6. Funk J. Early detection of glaucoma by longitudinal monitoring of the optic disc structure. Graefes Arch

Clin Exp Ophthalmol 1991;229:57-61. 1981;99:1992-4. trial determines that topical ocular hypotensive medication delays or prevents the onset of primary open-angle glaucoma. Arch Ophthalmol 2002;120:701-13.

7. Sommer A, Katz J, Quigley HA, et al. Clinically detectable nerve fiber atrophy precedes the onset of glaucomatous field loss. Arch Ophthalmol 1991;109:77-83.

8. Motolko M, Drance SM. Features of the optic disc in preglaucomatous eyes. Arch Ophthalmol

9. Kass MA, Heuer DK, Higginbotham EJ, et al. The Ocular Hypertension Treatment Study: A randomized

10. Zeimer R, Asrani S, Zou S, et al. Quantitative detection of glaucomatous damage at the posterior pole by 
Macular GCL Imaging in Preperimetric Glaucoma. Nakano, et al._Page 25

$1 \quad$ retinal thickness mapping. A pilot study. Ophthalmology 1998;105:224-31.

2 11. Giovannini A, Amato G, Mariotti C. The macular thickness and volume in glaucoma: an analysis in normal and glaucomatous eyes using OCT. Acta Ophthalmol Scand Suppl 2002;236:34-6.

12. Guedes V, Schuman JS, Hertzmark E, et al. Optical coherence tomography measurement of macular and nerve fiber layer thickness in normal and glaucomatous human eyes. Ophthalmology 2003;110:177-89.

13. Lederer DE, Schuman JS, Hertzmark E, et al. Analysis of macular volume in normal and glaucomatous eyes using optical coherence tomography. Am J Ophthalmol 2003;135:838-43.

14. Greenfield DS, Bagga H, Knighton RW. Macular thickness changes in glaucomatous optic neuropathy detected using optical coherence tomography. Arch Ophthalmol 2003;121:41-6.

15. Wollstein G, Schuman JS, Price LL, et al. Optical coherence tomography (OCT) macular and peripapillary retinal nerve fiber layer measurements and automated visual fields. Am J Ophthalmol 2004;138:218-25.

16. Wollstein $\mathrm{G}$, Ishikawa $\mathrm{H}$, Wang J, et al. Comparison of three optical coherence tomography scanning areas for detection of glaucomatous damage. Am J Ophthalmol 2005;139:39-43.

17. Medeiros FA, Zangwill LM, Bowd C, et al. Evaluation of retinal nerve fiber layer, optic nerve head, and macular thickness measurements for glaucoma detection using optical coherence tomography. Am J Ophthalmol 2005;139:44-55.

18. Leung $\mathrm{CK}$, Chan WM, Yung WH, et al. Comparison of macular and peripapillary measurements for the detection of glaucoma: An optical coherence tomography study. Ophthalmology. 2005;112:391-400. 
Macular GCL Imaging in Preperimetric Glaucoma. Nakano, et al._Page 26

20. Ishikawa H, Stein DM, Wollstein G, et al. Macular segmentation with optical coherence tomography. Invest Ophthalmol Vis Sci 2005;46:2012-7.

21. Tan O, Li G, Lu AT, et al. Mapping of macular substructures with optical coherence tomography for glaucoma diagnosis. Ophthalmology 2008;115:949-56.

22. Tan O, Chopra V, Lu AT, et al. Detection of macular ganglion cell loss in glaucoma by Fourier-domain optical coherence tomography. Ophthalmology 2009;116:2305-14.

23. Seong M, Sung KR, Choi $\mathrm{EH}$, et al. Macular and peripapillary retinal nerve fiber layer measurements by spectral domain optical coherence tomography in normal-tension glaucoma. Invest Ophthalmol Vis Sci $2010 ; 51: 1446-52$

24. Kotera Y, Hangai M, Hirose F, Mori S, Yoshimura N. Three-dimensional imaging of macular inner structures in glaucoma by using spectral-domain optical coherence tomography. Invest Ophthalmol Vis Sci. In press.

25. Sakamoto A, Hangai M, Nukada M, et al. Three-dimensional imaging of the macular retinal nerve fiber layer in glaucoma with spectral-domain optical coherence tomography. Invest Ophthalmol Vis Sci 2010;51:5062-70.

26. Hangai M, Yamamoto M, Sakamoto A, Yoshimura N. Ultrahigh-resolution versus speckle noise-reduction in spectral-domain optical coherence tomography. Opt Express 2009;17:4221-35.

27. Wollstein G, Paunescu LA, Ko TH, et al. Ultrahigh-resolution optical coherence tomography in glaucoma. Ophthalmology 2005;112:229-37. 
Macular GCL Imaging in Preperimetric Glaucoma. Nakano, et al._Page 27

1 28. Mumcuoglu T, Wollstein G, Wojtkowski M, et al. Improved visualization of glaucomatous retinal damage using high-speed ultrahigh-resolution optical coherence tomography. Ophthalmology 2008;115:782-9.

29. Schmitt JM, Xiang SH, Yung KM. Speckles in optical coherence tomography. J Biomed Optics 1999;4:95-105.

30. Sander B, Larsen M, Thrane L, et al. Enhanced optical coherence tomography imaging by multiple scan averaging. Br J Ophthalmol 2005 ;89:207-12.

31. Sakamoto A, Hangai M, Yoshimura N. Spectral-domain optical coherence tomography with multiple B-scan averaging for enhanced imaging of retinal diseases. Ophthalmology 2008;115:1071-8.

32. Byeon $\mathrm{SH}$, Chu YK, Lee H, et al. Foveal ganglion cell layer damage in ischemic diabetic maculopathy. density of the primate retina. Nature 1989;341:643-6.

33. Ooto S, Hangai M, Sakamoto A, et al. Three-dimensional profile of macular retinal thickness in normal Japanese eyes. Invest Ophthalmol Vis Sci 2010;51:465-73.

34. Mori S, Hangai M, Sakamoto A, Yoshimura N. Spectral-domain optical coherence tomography measurement of macular volume for diagnosing glaucoma. J Glaucoma. 2010;19:528-34.

35. Van Buren JM. The Retinal Ganglion Cell Layer. Springfield, IL: Charles C. Thomas; 1963.

36. Wassle H, Grunert U, Rohrenbeck J, Boycott BB. Cortical magnification factor and the ganglion cell

37. Curcio CA, Allen KA. Topography of ganglion cells in human retina. J Comp Neurol 1990;300:5-25. 
Macular GCL Imaging in Preperimetric Glaucoma. Nakano, et al.-Page 28

1 Re-revised Table 1 Mean Macular Retinal Layer Thicknesses Profile Measured on Vertical and Horizontal

2 Scans in Eyes with and without Glaucoma

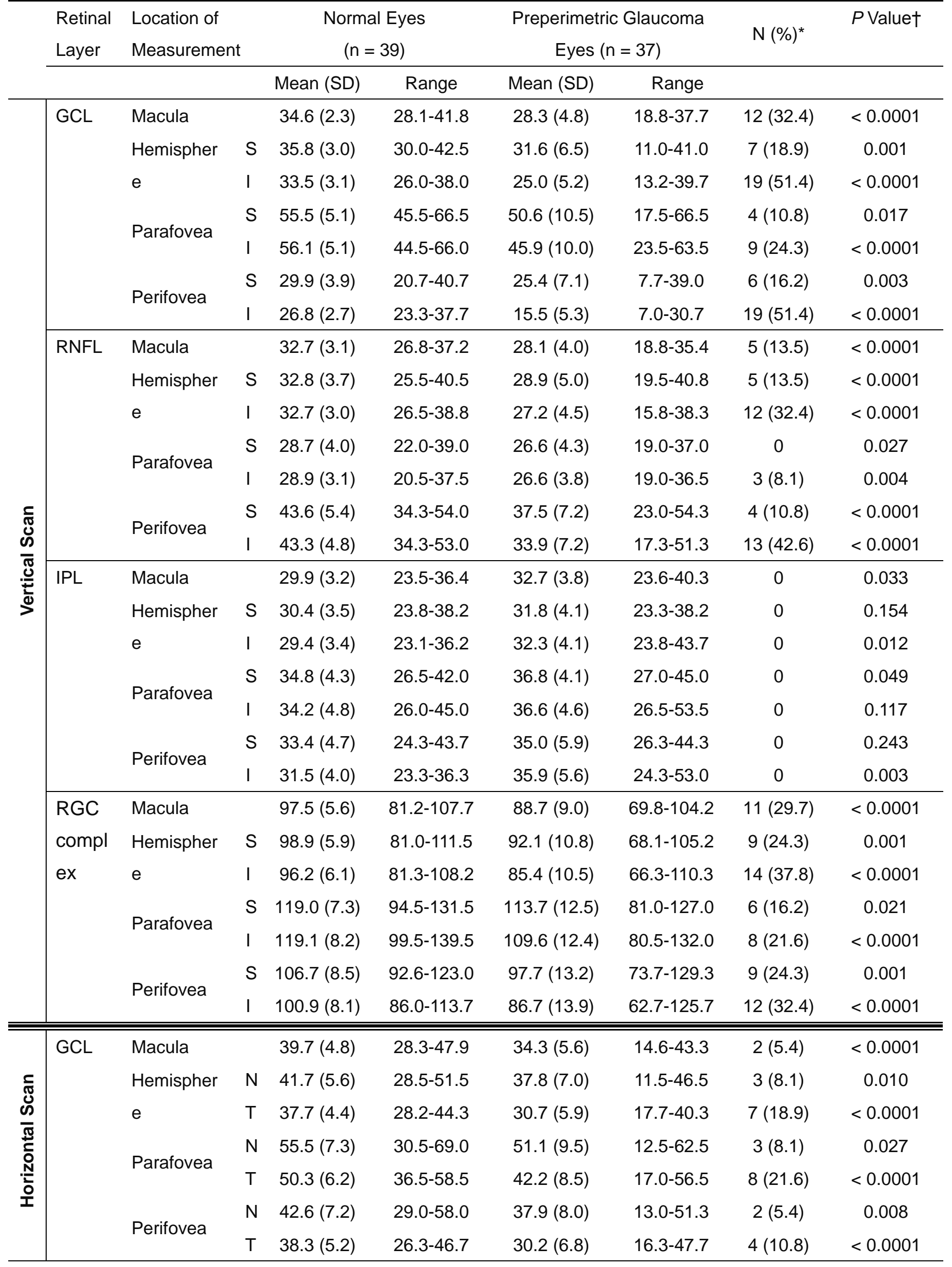


Macular GCL Imaging in Preperimetric Glaucoma. Nakano, et al._Page 29

\begin{tabular}{|c|c|c|c|c|c|c|c|c|}
\hline \multirow[t]{7}{*}{ RNFL } & \multicolumn{2}{|l|}{ Macula } & \multirow{2}{*}{$\begin{array}{l}22.1(1.9) \\
29.9(3.2)\end{array}$} & \multirow{2}{*}{$\begin{array}{l}17.1-25.4 \\
22.7-36.5\end{array}$} & \multirow{2}{*}{$\begin{array}{l}20.8(1.7) \\
28.3(3.2)\end{array}$} & \multirow{2}{*}{$\begin{array}{l}18.2-25.6 \\
21.8-34.2\end{array}$} & \multirow{2}{*}{$\begin{array}{l}0 \\
0\end{array}$} & \multirow{2}{*}{$\begin{array}{l}0.002 \\
0.037\end{array}$} \\
\hline & Hemispher & $\mathrm{N}$ & & & & & & \\
\hline & e & $\mathrm{T}$ & $14.3(1.6)$ & $11.5-17.5$ & $13.8(1.8)$ & $10.7-17.5$ & 0 & 0.012 \\
\hline & \multirow{2}{*}{ Parafovea } & $\mathrm{N}$ & $20.4(2.3)$ & 13.0-23.9 & $19.7(2.4)$ & 14.1-23.9 & 0 & 0.165 \\
\hline & & $\mathrm{T}$ & $15.4(2.0)$ & $12.5-19.6$ & $14.8(1.9)$ & $11.0-18.5$ & 0 & 0.196 \\
\hline & \multirow{2}{*}{ Perifovea } & $\mathrm{N}$ & $44.0(5.3)$ & $34.7-56.7$ & $41.5(5.5)$ & $28.7-50.3$ & $2(5.4)$ & 0.043 \\
\hline & & $\mathrm{T}$ & $16.1(2.2)$ & $12.0-21.3$ & $14.6(2.8)$ & $9.7-20.3$ & $1(2.7)$ & 0.009 \\
\hline \multirow[t]{7}{*}{ IPL } & Macula & & $30.0(2.6)$ & 25.1-33.8 & $30.4(2.3)$ & $25.8-34.4$ & 0 & 0.493 \\
\hline & Hemispher & $\mathrm{N}$ & $30.4(2.9)$ & $23.5-35.7$ & $31.1(2.8)$ & 25.3-36.2 & 0 & 0.249 \\
\hline & e & $\mathrm{T}$ & $29.6(2.8)$ & $24.5-34.5$ & $29.7(2.4)$ & $23.5-34.0$ & 0 & 0.996 \\
\hline & \multirow{2}{*}{ Parafovea } & $\mathrm{N}$ & $33.1(3.3)$ & $25.2-38.1$ & $34.5(3.7)$ & $26.0-41.5$ & 0 & 0.099 \\
\hline & & $\mathrm{T}$ & $32.2(3.0)$ & $25.6-37.7$ & $33.2(3.1)$ & $26.5-41.1$ & 0 & 0.184 \\
\hline & \multirow{2}{*}{ Perifovea } & $\mathrm{N}$ & $34.9(4.1)$ & $26.7-43.7$ & $35.7(3.1)$ & $30.0-41.7$ & 0 & 0.326 \\
\hline & & $\mathrm{T}$ & $34.4(4.3)$ & $25.3-41.7$ & $33.6(3.1)$ & 26.3-38.2 & 0 & 0.354 \\
\hline \multirow{7}{*}{$\begin{array}{l}\text { RGC } \\
\text { compl } \\
\text { ex }\end{array}$} & Macula & & $91.8(5.3)$ & 78.3-100.8 & 85.7 (7.2) & $65.4-98.9$ & $8(21.6)$ & $<0.0001$ \\
\hline & Hemispher & $\mathrm{N}$ & $101.9(7.1)$ & $83.4-112.5$ & $97.2(10.0)$ & $67.0-115.5$ & $5(13.5)$ & 0.022 \\
\hline & e & $\mathrm{T}$ & $81.8(4.4)$ & $73.0-90.7$ & $74.1(7.2)$ & $56.3-96.3$ & 7 (18.9) & $<0.0001$ \\
\hline & \multirow{2}{*}{ Parafovea } & $\mathrm{N}$ & $109.1(9.2)$ & $80.5-125.4$ & $105.9(11.5)$ & $70.0-122.5$ & $2(5.4)$ & 0.182 \\
\hline & & $\mathrm{T}$ & $98.1(7.3)$ & $79.3-112.5$ & $90.7(10.1)$ & $66.0-124.5$ & $4(10.8)$ & 0.001 \\
\hline & \multirow{2}{*}{ Perifovea } & $\mathrm{N}$ & $121.5(9.0)$ & $99.7-136.3$ & $114.4(12.6)$ & $79.0-137.3$ & $4(10.8)$ & 0.007 \\
\hline & & $\mathrm{T}$ & $89.0(5.6)$ & $78.7-100.0$ & $78.8(8.7)$ & $57.3-97.7$ & 5 (13.5) & $<0.0001$ \\
\hline
\end{tabular}

* Number and percent of eyes with abnormal thinning in each macular layer at each macular region in eyes with preperimetric glaucoma. Macular thickness at each region were defined as "abnormal" when the mean thickness was abnormally thin (outside the lower $99 \%$ confidential interval confidential limit in the normal eyes).

† Unpaired $t$-test

$\mathrm{GCL}$ = ganglion cell layer; $\mathrm{I}=$ Inferior; $\mathrm{IPL}=$ inner plexiform layer; $\mathrm{RGC}=$ retinal ganglion cell; $\mathrm{RGC}$ complex $=\mathrm{RNFL}$ $+\mathrm{GCL}+\mathrm{IPL} ; \mathrm{RNFL}=$ retinal nerve fiber layer; $\mathrm{S}=$ Superior; $\mathrm{SD}=1$ standard deviation 
Macular GCL Imaging in Preperimetric Glaucoma. Nakano, et al._Page 30

1 Table 2. Sensitivity and Specificity for Detecting Abnormal Thinning of Macular Layer Structures and the

2 Circumpapillary Retinal Nerve Fiber Layer

\begin{tabular}{|c|c|c|c|c|c|}
\hline & & & & $\begin{array}{c}\text { No. of Eyes with } \\
\text { Abnormal Thinning } \\
\text { in PPG } \\
\text { (Sensitivity [\%]) } \\
\mathrm{n}=37\end{array}$ & $\begin{array}{c}\text { No. of Eyes with } \\
\text { Abnormal Thinning in } \\
\text { Normal Eyes } \\
\text { (Specificity [\%]) } \\
\mathrm{n}=35\end{array}$ \\
\hline & & & Vertical & $30(81.1)$ & $0(100.0)$ \\
\hline & & GCL & Horizontal & $15(40.5)$ & $1(97.1)$ \\
\hline & & & $\mathrm{V}$ or $\mathrm{H}$ & $31(83.8)$ & $1(97.1)$ \\
\hline & & & Vertical & $18(48.6)$ & $1(97.1)$ \\
\hline & & RNFL & Horizontal & $8(21.6)$ & $2(94.3)$ \\
\hline (Alorme & 1030 & & V or H & $21(56.8)$ & $3(91.4)$ \\
\hline ctudy) & 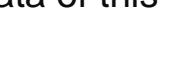 & & Vertical & $2(5.4)$ & $0(100.0)$ \\
\hline & & IPL & Horizontal & $2(5.4)$ & $0(100.0)$ \\
\hline & & & Vor $\mathrm{H}$ & $4(10.8)$ & $0(100.0)$ \\
\hline & & $\mathrm{BGC}$ & Vertical & $22(59.5)$ & $0(100.0)$ \\
\hline & & (1) & Horizontal & $18(48.6)$ & $2(94.3)$ \\
\hline & & & $\mathrm{V}$ or $\mathrm{H}$ & $26(70.3)$ & 2 (94.3) \\
\hline cpRNFL & Instrumer & ntal normat & e database & $16(43.2)$ & $2(94.3)$ \\
\hline (Spectralis) $^{\dagger}$ & Normal ey & ye data of & is study & $20(54.1)$ & $2(94.3)$ \\
\hline $\begin{array}{l}\mathrm{cpRNFL} \\
(\mathrm{GDx}-\mathrm{VCC})^{\ddagger}\end{array}$ & Instrumer & ntal Norma & ve database & $9(24.3)$ & $1(97.1)$ \\
\hline
\end{tabular}

*Macular thickness parameters were defined as "abnormal" when the mean thickness was abnormally thin (outside the lower $99 \%$ confidential interval $[\mathrm{Cl}]$ limit in our normal eyes) in at least one $0.5-\mathrm{mm}$ segment of the macula along vertical or horizontal scan.

${ }^{\dagger} \mathrm{cpRNFL}$ on Spectralis was defined as "abnormal" when the mean thickness of the whole cpRNFL or at least one sector of cpRNFL was abnormally thin (outside the lower $99 \% \mathrm{Cl}$ limit for the mean of the instrumental normative database or the normal eyes).

${ }^{\ddagger} \mathrm{CpRNFL}$ on GDx-VCC was defined as "abnormal" when the mean values of at least one thickness parameters among "NFI," "TSNIT average," "superior average," "inferior average," or "TSNIT Std. Dev." were abnormal (outside the lower $99 \% \mathrm{Cl}$ limit for the mean of the instrumental normative database).

$\mathrm{cpRNFL}=$ circumpapillary retinal nerve fiber layer; $\mathrm{GCL}=$ ganglion cell layer; $\mathrm{H}=$ horizontal; $\mathrm{IPL}=$ inner plexiform layer; $P P G=$ preperimetric glaucoma; $R G C=$ retinal ganglion cell; $R G C$ complex = $\mathrm{RNFL}+\mathrm{GCL}+\mathrm{IPL} ; \mathrm{V}=$ vertical; VCC = variable corneal compensation 
Macular GCL Imaging in Preperimetric Glaucoma. Nakano, et al.-Page 31

$1 \quad$ Figure Legends

2 Figure 1. Speckle-noise-reduced spectral-domain optical coherence tomography (SD-OCT) B-scans

3 through the central fovea in a normal eye. A, B, Horizontal (A) and vertical (B) 9-mm scans show clearly

4 distinguishable layers. C, Magnified view of inferior macular region in (B) showing ganglion cell layer (GCL),

5 retinal nerve fiber layer (RNFL), and inner plexiform layer (IPL). The double-headed arrows indicate various

6 distances from the central fovea (zero); the segment from 0 to $0.5 \mathrm{~mm}$ is considered to be within the fovea,

$7 \quad 0.5$ to $1.5 \mathrm{~mm}$ is considered the parafoveal region, and 1.5 to $3.0 \mathrm{~mm}$ is considered the perifoveal segment.

8 The shape of the GCL and IPL appears vertically and horizontally symmetrical and that of RNFL appears to

9 be only vertically symmetrical.

11 Figure 2. Images showing effects of speckle-noise-reduction by multiple B-scan averaging of

12 spectral-domain optical coherence tomography (SD-OCT) B-scans in a normal eye. A, B, Single B-scan

13 image. C,D, Average of 10 B-scans at the same location. E,F, Average of 50 B-scans at the same location.

14 B,D,F, Magnified (4X) views of areas outlined by red dashed lines in A, C, and E show that averaging more

15 scans results in greater reduction in speckle noise and increasing clarification of the boundaries between the

16 retinal nerve fiber layer (RNFL), ganglion cell layer (GCL), and inner plexiform layer (IPL).

18 Figure 3. Mean macular ganglion cell layer (GCL) thickness in normal eyes as a function of distance from

19 the central fovea along the horizontal (A-C) and vertical (D-F) ocular meridians. Mean macular GCL

20 thicknesses in the nasal (A), temporal (B), superior (D), and inferior (E) hemispheres as measured by 2

21 independent delineators were superimposed. Mean arbitrated macular GCL thickness of the nasal-temporal 
Macular GCL Imaging in Preperimetric Glaucoma. Nakano, et al._Page 32

1 (C), and superior-inferior (F) hemispheres were superimposed. Arbitration was performed by group review

2 and discussion among the 2 delineators and a third observer. In the horizontal meridian (A), the gap in the

3 nasal curve $3.5 \mathrm{~mm}$ from the center of the fovea represents the site of the optic nerve head. ${ }^{*} P<0.0001 ;{ }^{* *} P$

$4<0.001 ;{ }^{* \star *} P<0.05$

6 Figure 4. Superimposed graphs for the mean thickness of the macular ganglion cell layer (GCL); data for

7 eyes with preperimetric glaucoma was superimposed on that for normal eyes as a function of distance from

8 the central fovea along the vertical and horizontal ocular meridians. The mean thickness of the macular GCL

9 in the superior (A), inferior (B), nasal (C), and temporal (D) hemispheres arbitrated by group review and

10 discussion among the 2 delineators and a third observer are plotted. ${ }^{*} P<0.0001 ;{ }^{* *} P<0.001 ;{ }^{* *} P<0.05$

12 Figure 5. Histograms showing percent thickness of the macular ganglion cell layer $(\mathrm{GCL})(\mathbf{A}, \mathbf{B})$, macular

13 retinal nerve fiber layer $(\mathrm{RNFL})(\mathbf{C}, \mathbf{D})$, macular inner plexiform layer (IPL) $(\mathbf{E}, \mathbf{F})$, and retinal ganglion cell

14 complex $(\mathrm{RGC}$ complex $=\mathrm{RNFL}+\mathrm{GCL}+\mathrm{IPL})$ in the macula $(\mathbf{G}, \mathbf{H})$ on vertical $(\mathbf{A}, \mathbf{C}, \mathbf{E}, \mathbf{G})$ and horizontal

15 (B,D,F,H) 9-mm speckle-noise-reduced spectral-domain optical coherence tomography (SD-OCT) B-scans

16 in eyes with preperimetric glaucoma $(n=37)$ compared to mean thickness of these layers in normal eyes

17 (n=39). A-1, C-1, E-1, G-1, Results for the macular, inferior macular, and superior macular regions. A-2, C-2,

18 E-2, F-2, Results for the inferior and superior perifoveal regions (1.5-3.0 $\mathrm{mm}$ from the center of the fovea)

19 and for the inferior and superior parafoveal regions $(0.5-1.5 \mathrm{~mm}$ from the center of the fovea). B-1, D-1, F-1,

$20 \mathbf{H - 1}$, Results for the macular, nasal macular, and temporal macular regions. B-2, D-2, F-2, H-2, Results for

21 the nasal and temporal perifoveal and parafoveal regions. 
Macular GCL Imaging in Preperimetric Glaucoma. Nakano, et al._Page 33

2 Figure 6. Macular ganglion cell layer (GCL) thinning visible on radial speckle-noise-reduced

3 spectral-domain optical coherence tomography (SD-OCT). The patient was a 45-year-old woman and

4 intraocular pressure in the left eye was $14-16 \mathrm{mmHg}$ without glaucoma medication. On color disc $(\mathbf{A})$ and

5 fundus (B) photographs of this patient's left eye, localized thinning of the neuroretinal rim of the optic nerve

6 head (white arrow) and wedge-shaped retinal nerve fiber layer (RNFL) defects (white arrowheads) are

7 visible. C, This infrared image of the right eye shows the directions of radial 9-mm speckle-noise-reduced

8 SD-OCT B-scans of the right eye shown in E-L. The red portions of the yellow arrows indicate where the

9 ganglion cell layer (GCL) appears thinned on the SD-OCT images (inferior hemisphere) and blue portions of

10 the arrows indicate the equivalent regions in the superior hemisphere. D, Humphrey 24-2 SITA standard

11 pattern deviation map. E, Vertical SD-OCT B-scans through the fovea. Localized GCL thinning (red

12 double-headed arrows) is evident in the inferior hemisphere (left side of the image) but not in the superior

13 hemisphere (blue double-headed arrow indicates the comparable distance from the central fovea). F, G,

14 Magnified (2X) view of areas outlined by red dashed lines in $\mathbf{E}$. The red dashed lines in G represent the

15 boundaries of the macular GCL. RNFL = retinal nerve fiber layer; IPL = inner plexiform layer. Thinning of the

16 RNFL is associated with GCL atrophy. The inner plexiform layer (IPL) appears to be elevated where the

17 GCL is thinned, but it does not appear atrophic. H-L, Radial SD-OCT B-scans centered on the central fovea.

18 The GCL is noticeably thinned in a segment of the inferior hemisphere (K,L; red double-headed arrows)

19 compared to the equivalent area of the superior hemisphere $(\mathbf{I}, \mathbf{H}$; blue double-headed arrows). 
Macular GCL Imaging in Preperimetric Glaucoma. Nakano, et al._Page 34

$1 \quad$ Figure 7. Manual delineation of retinal layer boundaries $(\mathbf{A})$ and macular retinal layer thickness

2 measurements calculated by SD-OCT software by using vertical B-scans through the central fovea of the

3 right eye (shown in Figure 6). A, Red boundary lines delineate the retinal nerve fiber layer (RNFL), ganglion

4 cell layer (GCL), and inner plexiform layer (IPL) and were used for calculations of RNFL, GCL, and IPL

$5 \quad$ thickness at various distances from the central fovea to the periphery. B-E, Thickness curves (black lines)

6 for the RNFL, GCL, IPL, and the retinal ganglion cell complex (RGC complex = RNFL+GCL+IPL) in this eye,

7 which were generated from thickness calculations at various distances from the central fovea (negative

8 numbers on the $\mathrm{X}$-axis represent distances from the central fovea in the inferior hemisphere; positive

9 numbers represent distances in the superior hemisphere). Colored graphs were drawn using the mean

10 (green line) and confidence intervals of mean thicknesses measured in 39 normal Japanese eyes that were

11 examined at Kyoto University Hospital. The sector maps in the right column of $(\mathbf{B})-(\mathbf{E})$ indicate the mean

12 thickness within every $0.5-\mathrm{mm}$ segment from the central fovea. The red double-headed arrows indicate the

13 thinning of each layer as compared to its mean thickness in normal eyes. Red horizontal lines indicate

14 equivalent points in the superior and inferior hemispheres. The GCL (C) is greatly thinned in the inferior

15 hemisphere as compared to the mean thickness at an equivalent location in the superior hemisphere and as

16 compared to the mean thickness in normal eyes in this study (green line). Compared to normal eyes, the

$17 \mathrm{GCL}$ in this eye is abnormally thinned in 3 inferior segments from 1.5 to $3.0 \mathrm{~mm}$. The RNFL thinning in this

18 eye (black line in $\mathbf{B}$ ) appears to be milder compared to GCL thinning and borderline thinning is observed in 2

19 inferior segments from 2.0 to $3.0 \mathrm{~mm}$. The IPL thickness is within normal limits in all segments. Abnormal

20 thinning of the RGC complex (E) was detected in the 3 inferior segments from 1.5 to $3.0 \mathrm{~mm}$. F,G,

21 Automated measurement of circumpapillary RNFL (cpRNFL) thickness by using built-in software. F, Red 
Macular GCL Imaging in Preperimetric Glaucoma. Nakano, et al._Page 35

1 boundary lines delineate the cpRNFL. G, The cpRNFL thickness map in the left column shows the mean

2 (green line) and confidence intervals of mean thickness in the instrumental normative database. The sector

3 map in the right column indicates the mean cpRNFL thickness within each sector and the whole. The color

4 coding is as follows: purple, abnormal thickening (values higher than the upper $99 \%$ confidence interval [Cl]

$5 \quad$ limit for the mean in the 39 normal eyes); red, abnormal thinning (values lower than the lower $99 \% \mathrm{Cl}$ limit

6 for the mean in normal eyes); blue, borderline thickened (values between the upper $95 \%$ and upper $99 \% \mathrm{Cl}$

7 limit for the mean in normal eyes); yellow, borderline thinned (values between the lower $95 \%$ and lower $99 \%$

$8 \mathrm{Cl}$ limit for the mean for normal eyes); and green, within normal limits (values between the upper and lower

$995 \% \mathrm{Cl}$ limit for the mean in the 39 normal eyes). The Y-axis indicates thickness values ( $\mu \mathrm{m})$.

11 Figure 8. Photographs, vertical 9-mm speckle-noise-reduced spectral-domain optical coherence

12 tomography (SD-OCT) B-scan images, and macular ganglion cell layer (GCL) thickness curves based on

13 manual SD-OCT measurements of 2 eyes with preperimetric glaucoma. A-C, Images of a normal eye, D-O,

14 Images of eyes with preperimetric glaucoma. A,D,J, Color disc photographs. G,M, Color fundus

15 photographs. White arrows indicate neuroretinal rim thinning in the optic nerve head, and white arrowheads

16 indicate wedge-shaped localized retinal nerve fiber layer defects. B,C,E,F,H,K,L,N, Vertical SD-OCT

17 B-scans. C, F, H, L, N, Magnified (2X) view of areas outlined by red dashed lines in (B), (E) and (K). The red

18 dashed lines in $\mathbf{H}$ and $\mathbf{N}$ represent the boundaries of the macular GCL. Localized thinning of the macular

19 GCL at various distances from the central fovea is evident in the inferior hemisphere (red arrows), compared

20 to an equivalent area in the superior hemisphere (blue arrows) and to an equivalent area in a normal

21 macular GCL. I, O, Graphs of mean thickness curves of the GCL on vertical scans of 2 eyes with 
Macular GCL Imaging in Preperimetric Glaucoma. Nakano, et al._Page 36

1 preperimetric glaucoma. The $\mathrm{X}$-axis indicates distances $(\mathrm{mm})$ from the central fovea in the inferior

2 hemisphere (left side; negative numbers) and superior hemisphere (right side; positive numbers). The Y-axis

3 indicates GCL thickness $(\mu \mathrm{m})$. Color-coded curves are based on measurements obtained for 39 normal

4 Japanese eyes examined at Kyoto University Hospital. The sector maps in the right column of (I) and (O)

$5 \quad$ indicate the mean thickness every $0.5 \mathrm{~mm}$ from the central fovea. The color coding is as follows: purple =

6 abnormal thickening (values above the upper 99\% confidence interval $[\mathrm{Cl}]$ limit for the mean in normal

$7 \quad$ eyes); red = abnormal thinning (values lower than the lower $99 \% \mathrm{Cl}$ limit for the mean in normal eyes); blue

$8=$ borderline thickening (values between the upper 95\% and upper $99 \% \mathrm{Cl}$ limit for the mean in normal

9 eyes); yellow = borderline thinning (values between the lower 95\% and lower $99 \% \mathrm{CI}$ limit for the mean in

10 normal eyes); and green = within normal limits (values between the upper $95 \%$ and lower $95 \% \mathrm{Cl}$ limit for the

11 mean in normal eyes). The macular GCL was abnormally thin in the inferior hemisphere in both eyes in

12 some segments where the GCL thickness (black line) decreased as compared to that in the superior

13 hemisphere (red horizontal line) and as compared to the mean in normal eyes (green line); red

14 double-headed arrows indicate this difference. 


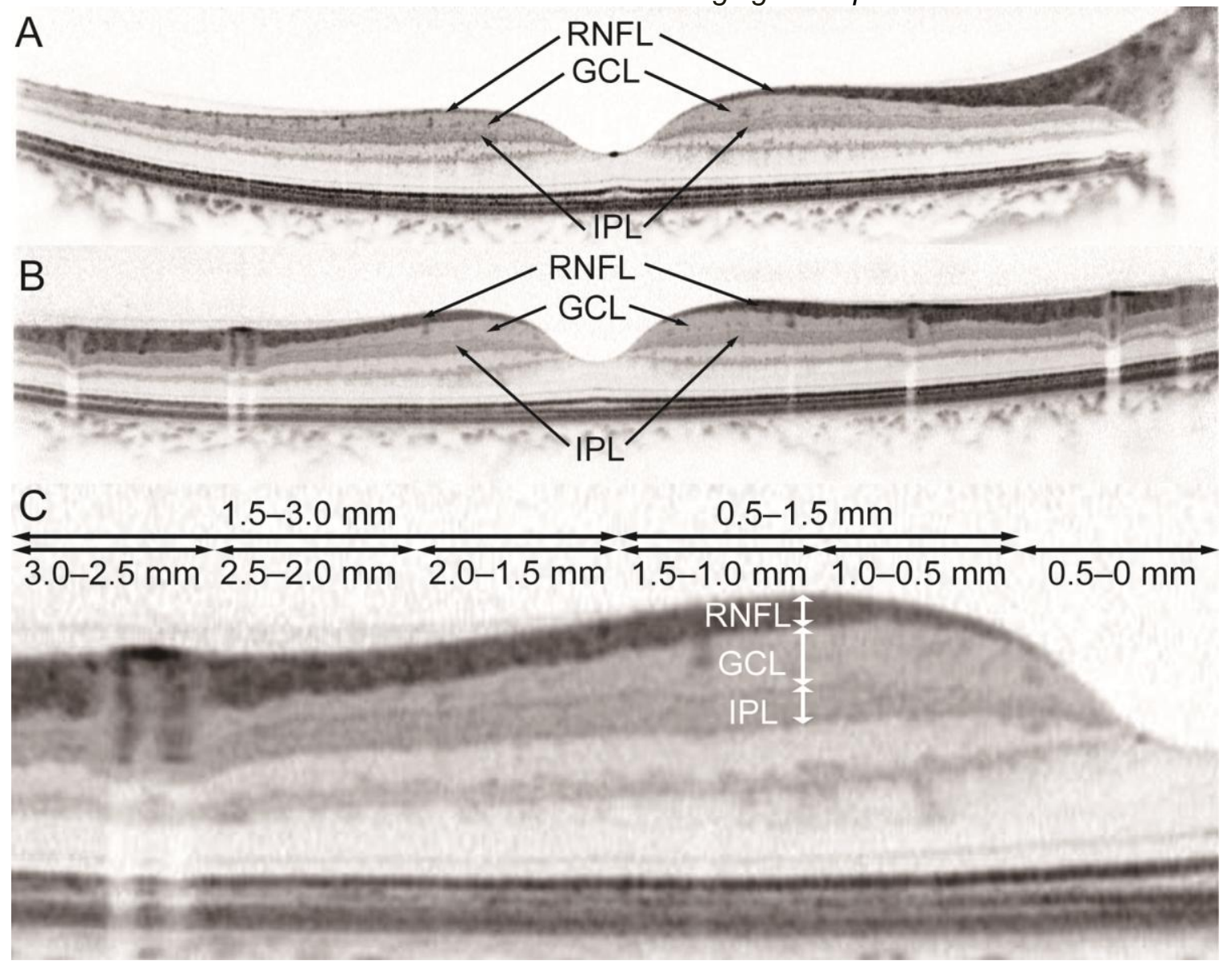

2 Figure 1 
Macular GCL Imaging in Preperimetric Glaucoma. Nakano, et al._Page 38

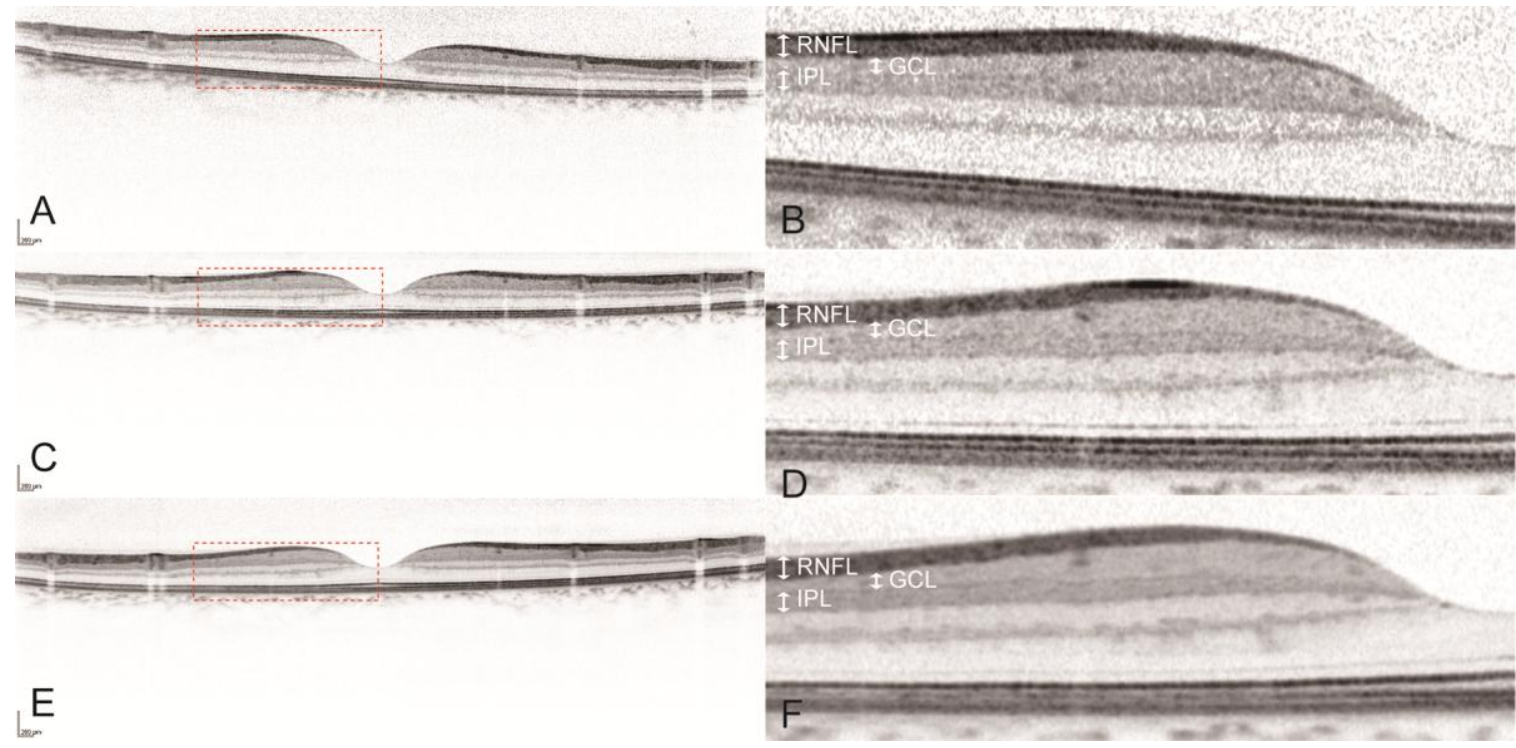

$1 \quad \mathrm{E}$

2 Figure 2 
Macular GCL Imaging in Preperimetric Glaucoma. Nakano, et al._Page 39
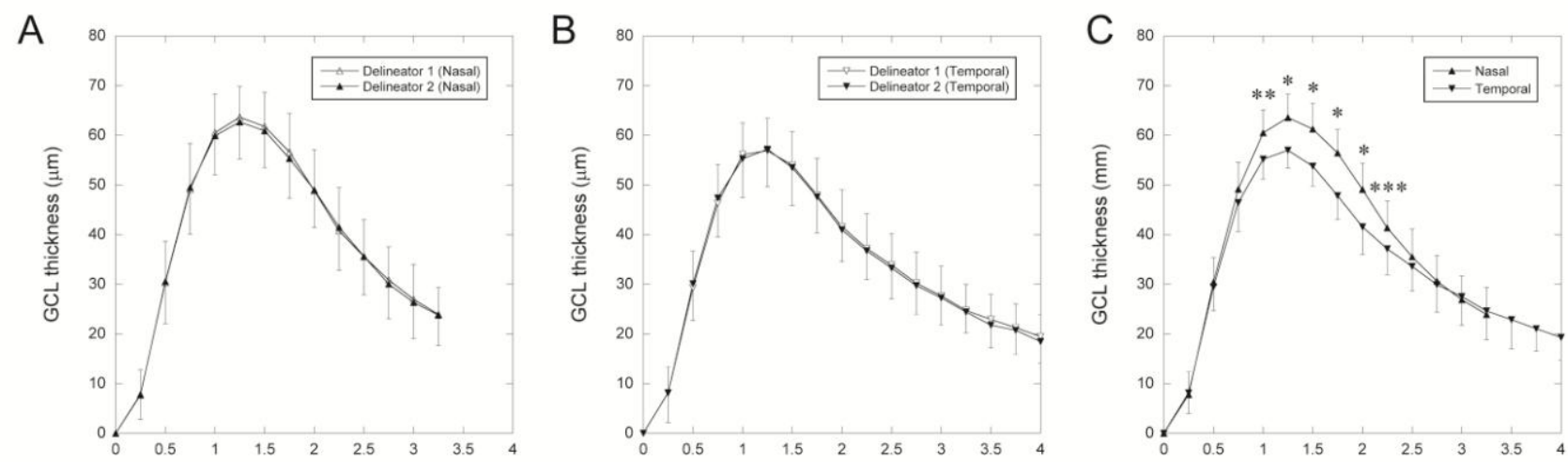

Distance from the center of the fovea $(\mathrm{mm})$

Distance from the center of the fovea $(\mathrm{mm})$

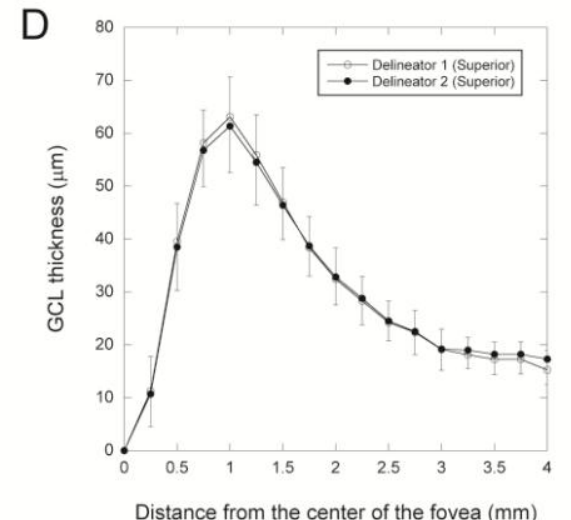

E

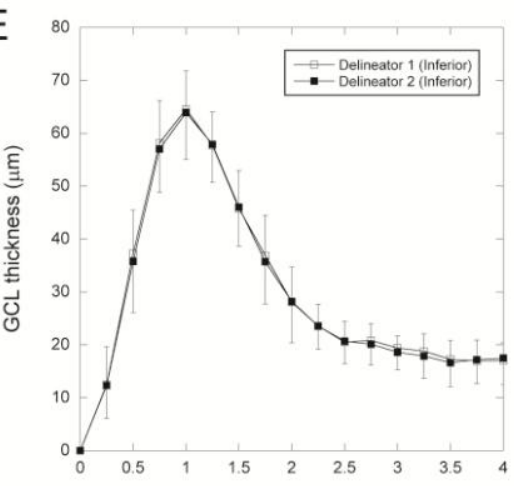

Distance from the center of the fovea $(\mathrm{mm})$

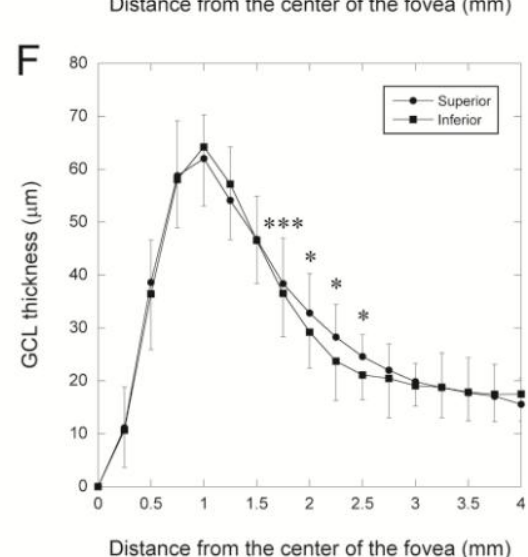

2 Figure 3 
Macular GCL Imaging in Preperimetric Glaucoma. Nakano, et al._Page 40

A

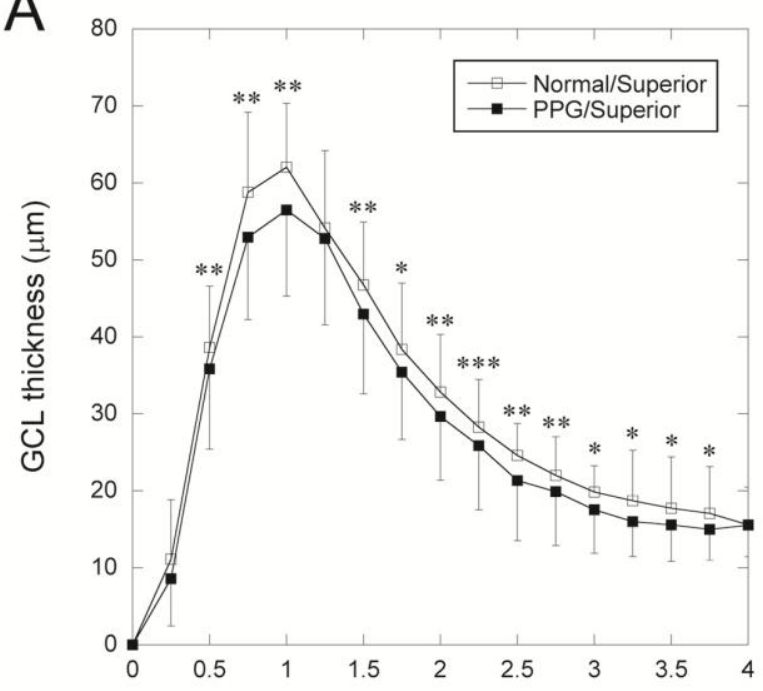

Distance from the center of the fovea $(\mathrm{mm})$

C

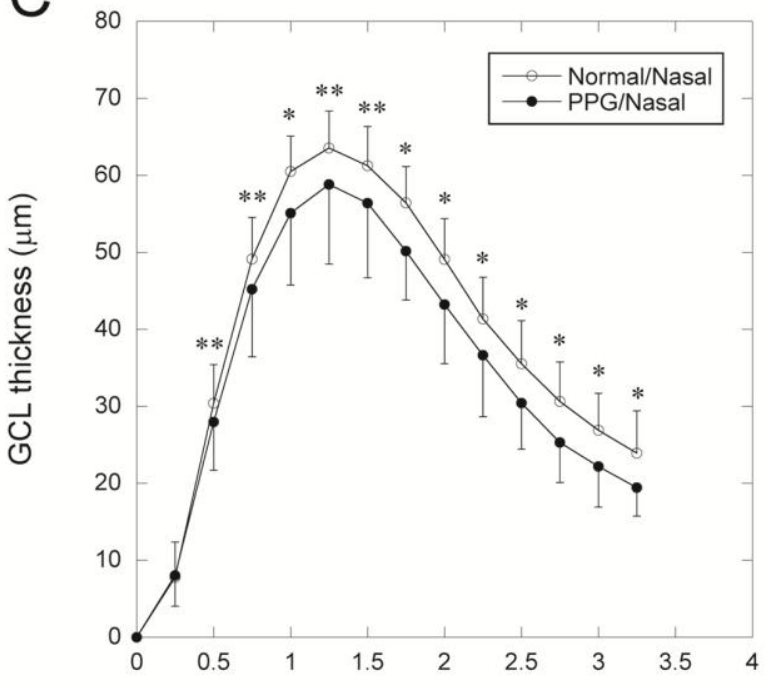

Distance from the center of the fovea $(\mathrm{mm})$

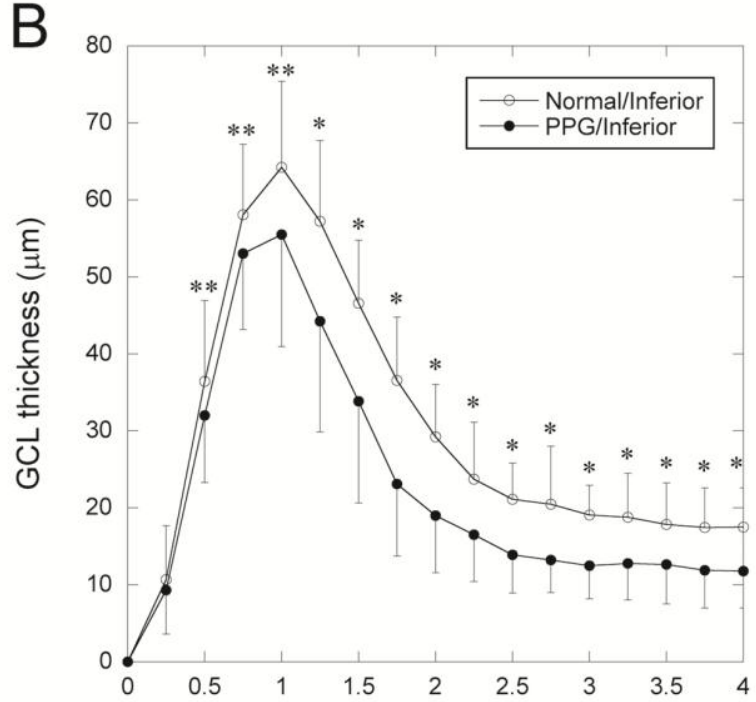

Distance from the center of the fovea $(\mathrm{mm})$

D

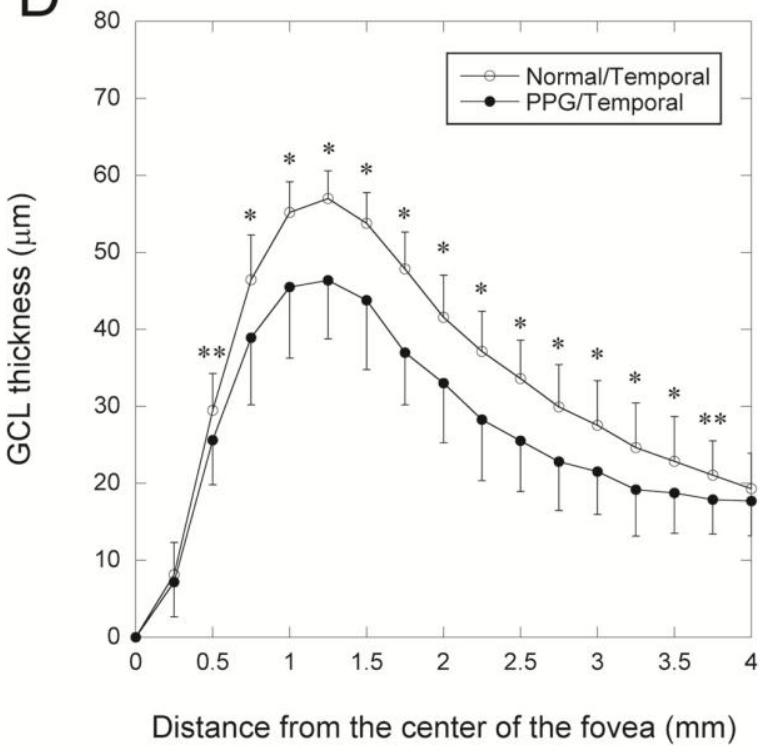


$\square$ Macula $\square$ Superior $(A, C, E, G)$ or nasal $(B, D, F, H)$ hemisphere $\quad$ Inferior $(A, C, E, G)$ or $(B, D, F, H)$ temporal hemisphere

$\square$ Superior $(A, C, E, G$ or nasal $(B, D, F, H)$ parafovea $\square$ Inferior $(A, C, E, G)$ or temporal $(B, D, F, H)$ parafovea $\square$ Superior $(A, C, E, G)$ or nasal $(B, D, F, H)$ perifovea $\square$ Inferior $(A, C, E, G)$ or temporal $(B, D, F, H)$ perifovea

A-1 10

A-2

B-1 18
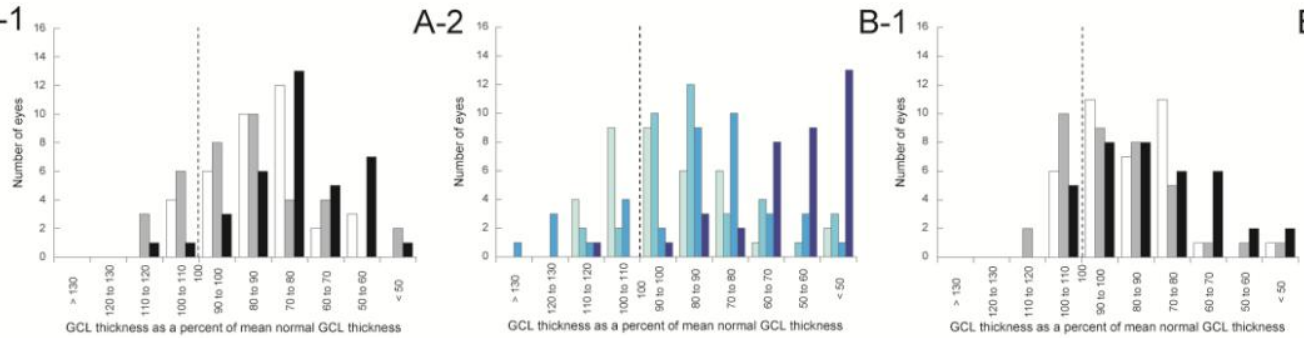

C-1 10

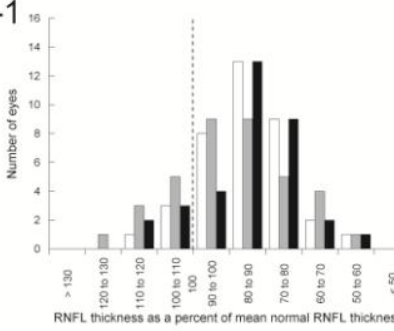

C-2
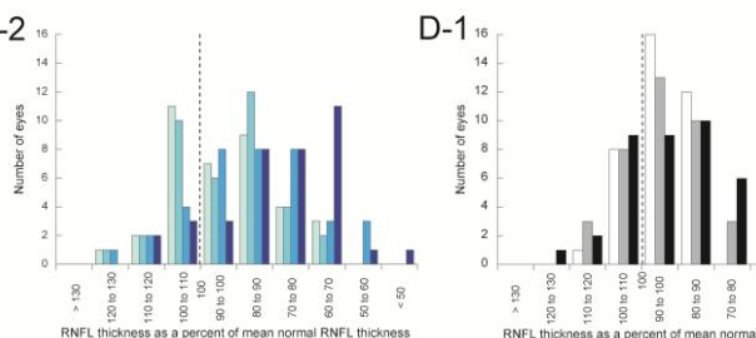

E-1

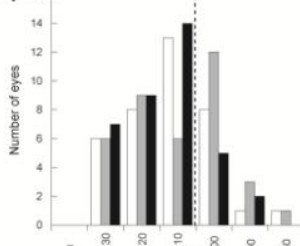

G-1

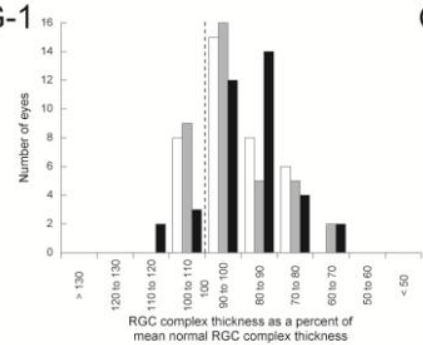

E-2

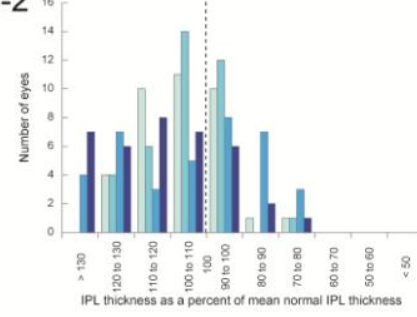

F-1

G-2
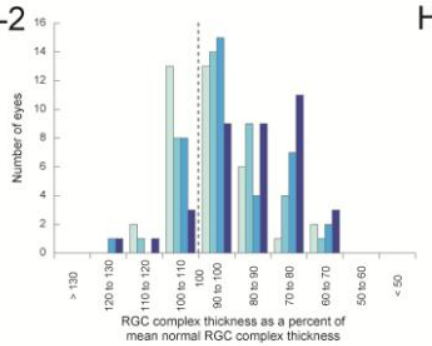

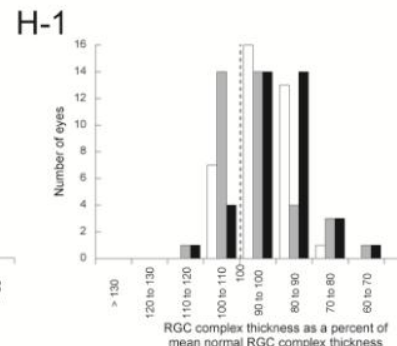

B-2 ,

D-2
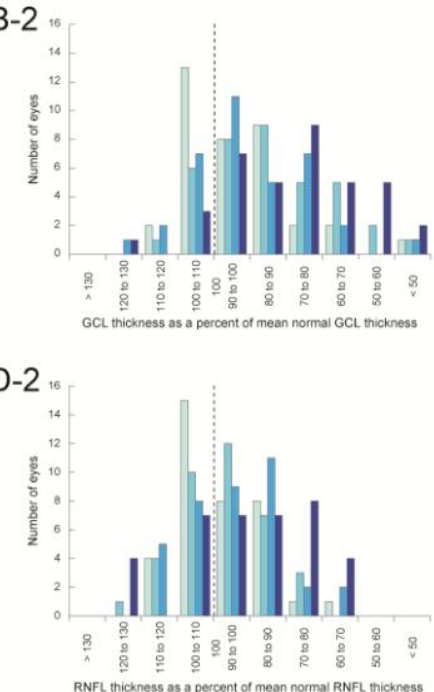

F-2

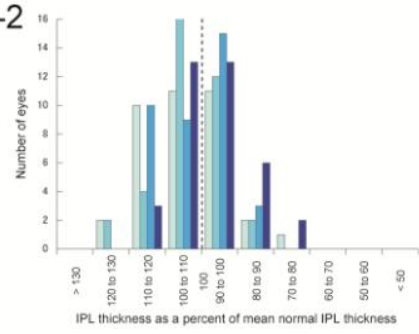

$\mathrm{H}-2$

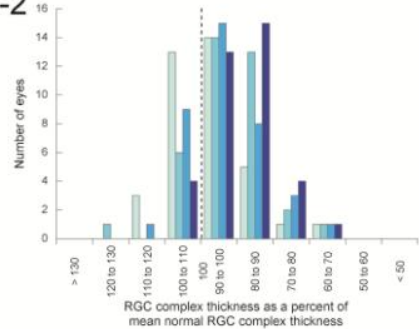

Figure 5 
Macular GCL Imaging in Preperimetric Glaucoma. Nakano, et al._Page 42
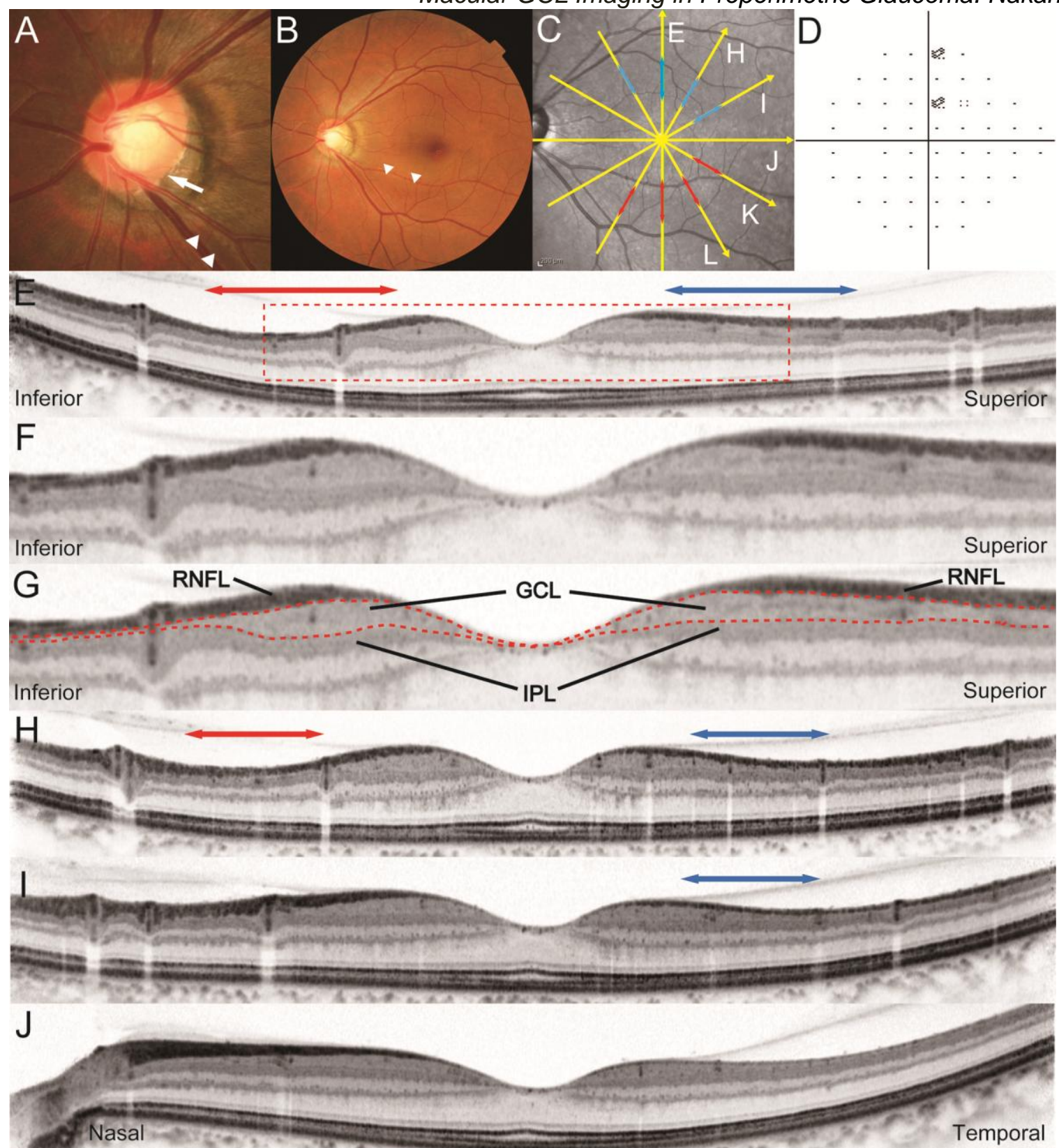

2 Figure 6 
Macular GCL Imaging in Preperimetric Glaucoma. Nakano, et al._Page 43
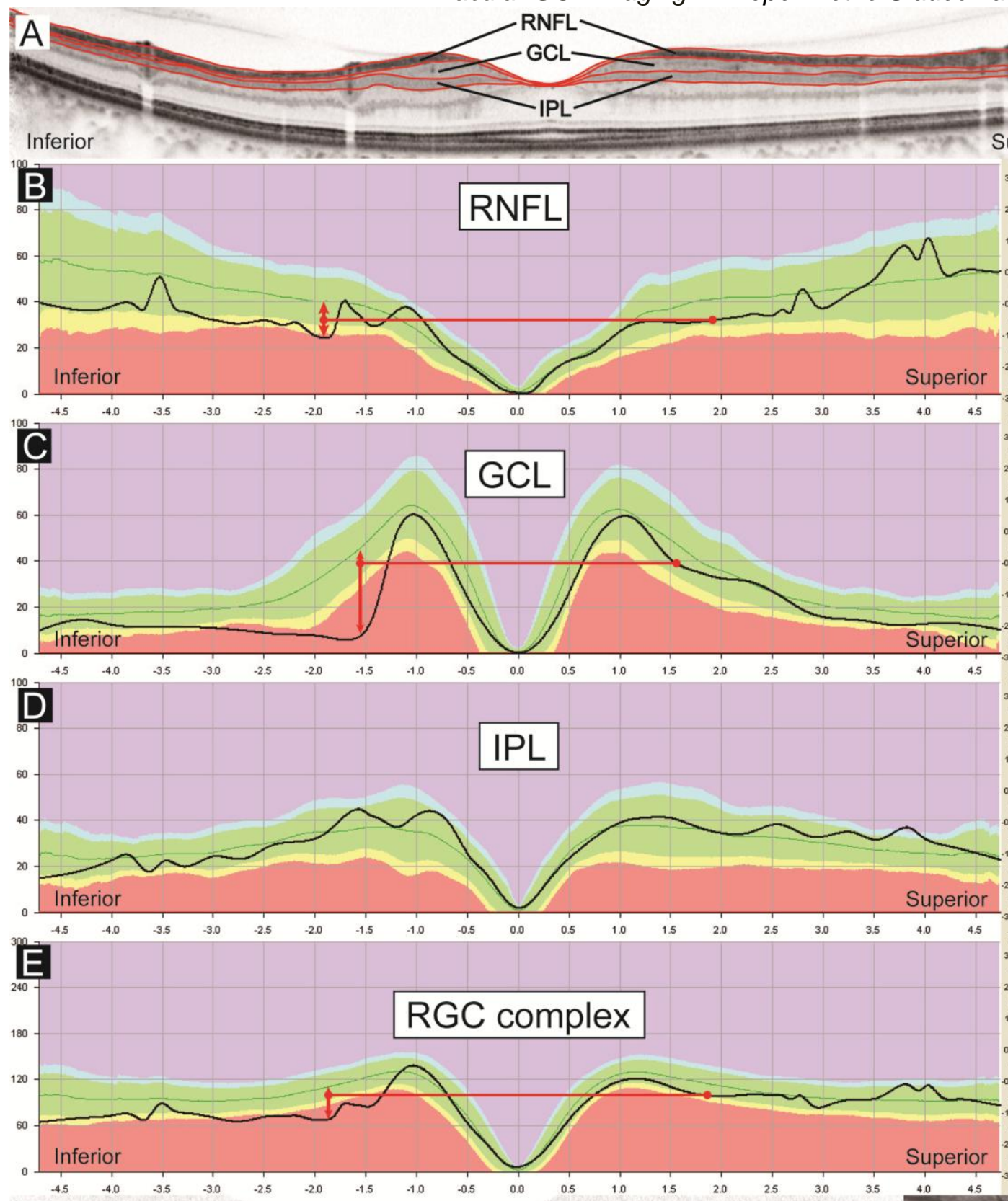

F

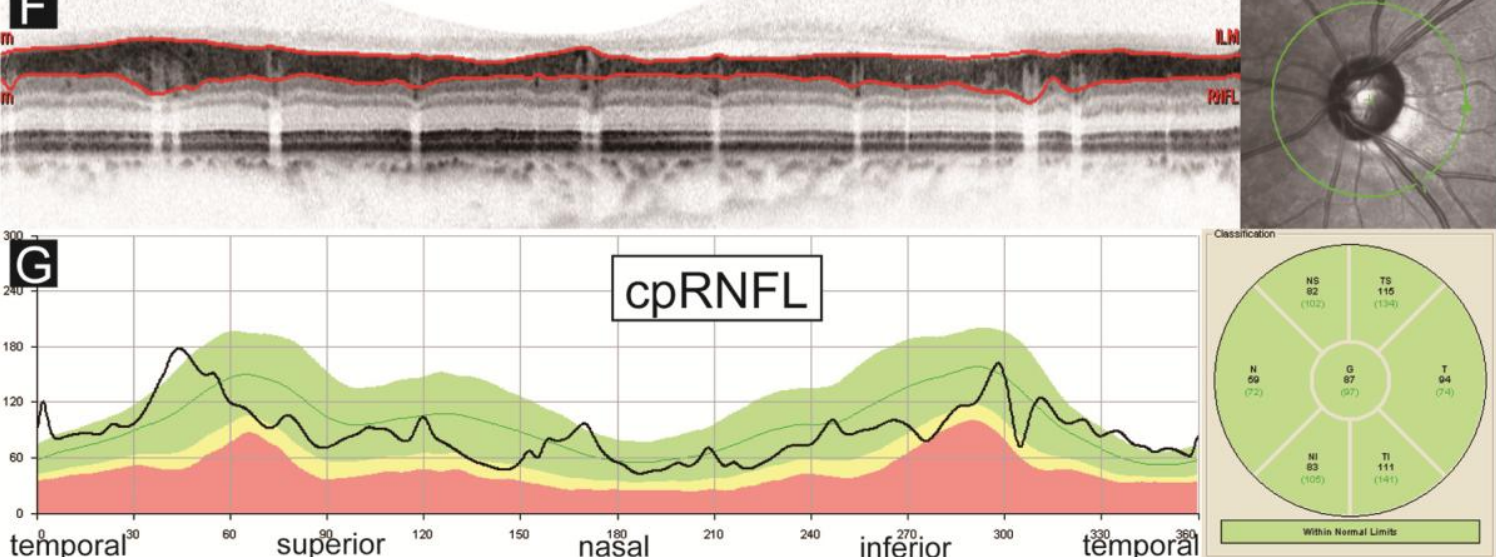

Figure 7 
Macular GCL Imaging in Preperimetric Glaucoma. Nakano, et al.-Page 44
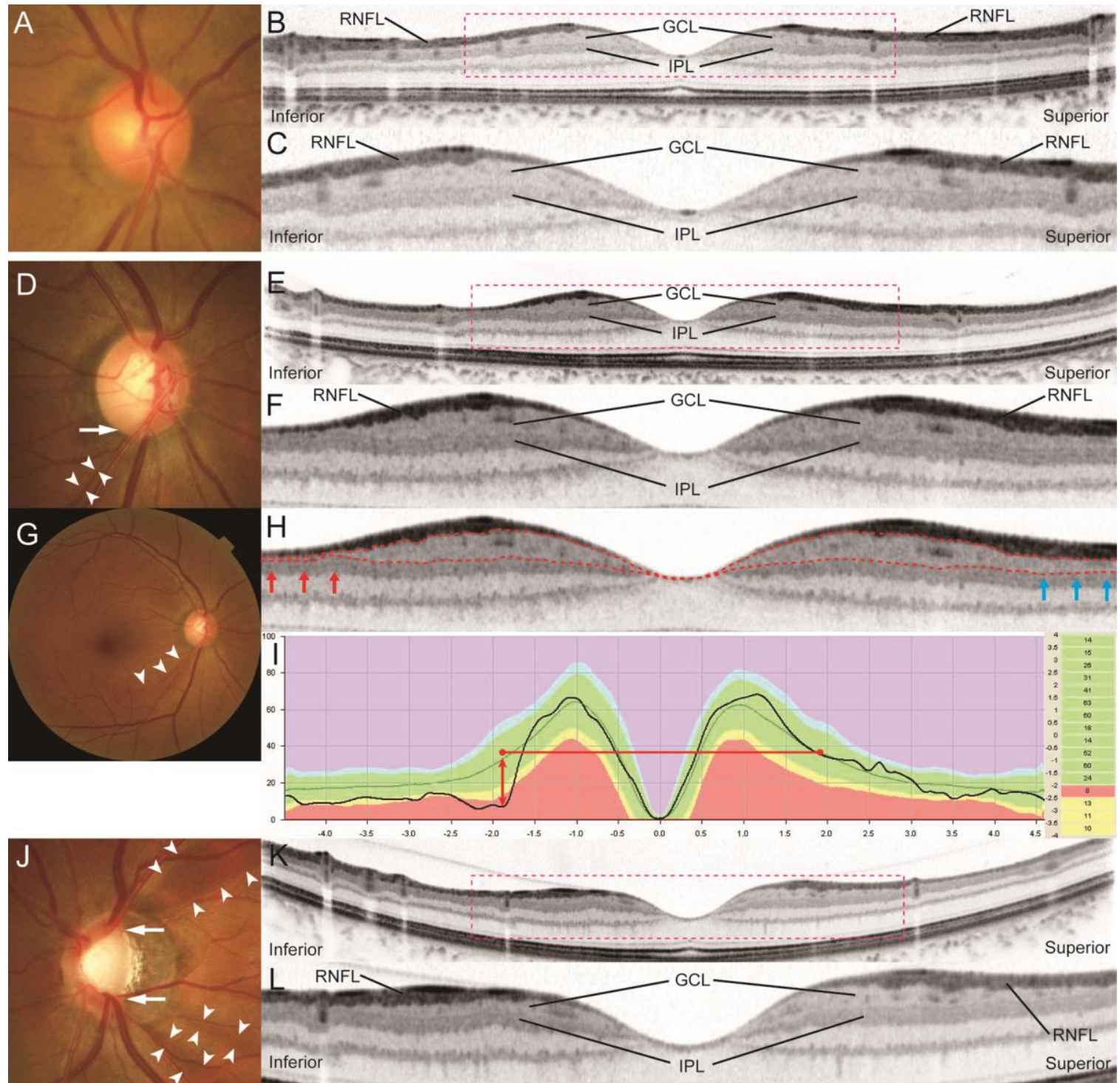

M
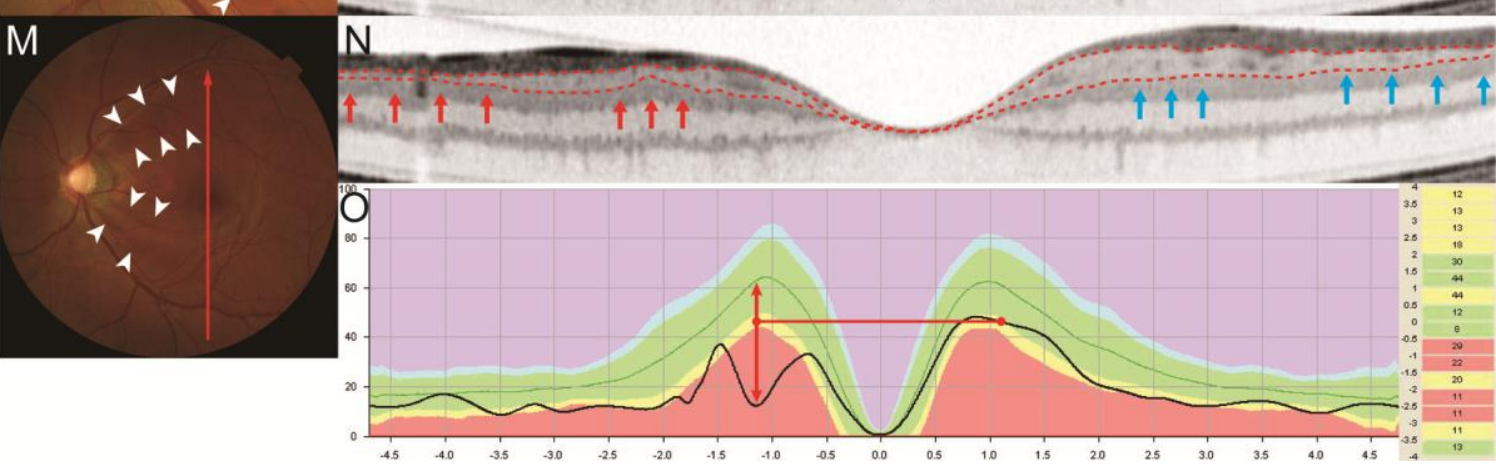

Figure 8 